\title{
Stakeholders' Perceptions of Factors Influencing the Use of Take-Home-Naloxone
}

\author{
Taylor J. Holland ${ }^{1}$, Jonathan Penm ${ }^{1,2}$, Jacinta Johnson ${ }^{3}$, , Maria Sarantou ${ }^{4}(\mathbb{D}$ \\ and Betty B. Chaar $1, *$ i \\ 1 School of Pharmacy, Faculty of Medicine and Health, The University of Sydney, \\ Camperdown, NSW 2006, Australia; thol5537@uni.sydney.edu.au (T.J.H.); \\ jonathan.penm@sydney.edu.au (J.P.) \\ 2 Department of Pharmacy, Prince of Wales Hospital, Randwick, NSW 2031, Australia \\ 3 UniSA Clinical and Health Sciences, University of South Australia, Adelaide, SA 5000, Australia; \\ Jacinta.Johnson@unisa.edu.au \\ 4 College of Medicine and Public Health, Flinders University, Bedford Park, SA 5042, Australia; \\ sara0044@flinders.edu.au \\ * Correspondence: betty.chaar@sydney.edu.au; Tel.: +61-2-9036-7101 or +61-425-210-547
}

Received: 9 June 2020; Accepted: 25 November 2020; Published: 3 December 2020

check for updates

\begin{abstract}
Background and Aims: Opioid associated death and overdose is a growing burden in societies all over the world. In recent years, legislative changes have increased access to naloxone in the take-home setting for use by patients with a substance use disorder and bystanders, to prevent opioid overdose deaths. However, few studies have explored the factors influencing the uptake by its multiple stakeholders. The aim of this scoping review was to explore the factors influencing the use of take-home naloxone from the perspectives of different stakeholders. Methods: A scoping review methodology was adopted with a systematic search of databases EMBASE, MEDLINE and PubMed. A variation of the search words "naloxone", "opioid" and "overdose" were used in each database. The articles were screened according to the predetermined inclusion/exclusion criteria and categorized based on their key perspective or target population. Results: The initial database search yielded a total of 1483 articles. After a series of screening processes, 51 articles were included for analysis. Two key stakeholder perspectives emerged: patients and bystanders $(n=36)$, and healthcare professionals $(\mathrm{n}=15)$. Within the patient and bystander group, a strong consensus arose that there were positive outcomes from increased access to take-home naloxone and relevant training programs. Despite these positive outcomes, some healthcare professionals were concerned that take-home naloxone would encourage high-risk opioid use. Conclusion: Take-home naloxone is slowly being introduced into community practice, with a sense of enthusiasm from patients and bystanders. There are still a number of barriers that need to be addressed from healthcare professionals' perspective. Future research should be aimed at emergency care professionals outside of the US, who are most experienced with naloxone and its potential impact on the community.
\end{abstract}

Keywords: take-home naloxone; opioid; overdose; injecting drug users (IDUs); opioid antagonist; patients' perspective; healthcare professional perspective

\section{Introduction}

Opioid overdose and misuse is a significant public health burden worldwide and is a common cause of drug-related deaths in Australia [1]. In 2012 in Australia, there was a total number of 564 accidental deaths from opioid overdose [2], almost half that of total road accident associated deaths [3]. The number of opioid-related deaths has been sharply rising with a 21-fold increase observed in the 
state of Victoria, Australia, caused by oxycodone from 2000 to 2009 [4]. This has been, in part, due to a 15-fold increase in opioid prescriptions dispensed through the Australian Pharmaceutical Benefits Scheme (PBS) from 1992 to 2012 [5], with oxycodone being the main contributor, rising from 35.3 to 89.2 per 1000 population between March 2002 and August 2007 [6].

The increase in use of opioids has occurred for a variety of reasons. One large factor can be attributed to the increased acceptance of opioids for pain treatment, as they were once considered safe, with low risk of iatrogenic addiction [7]. However, recent trends show that rates of iatrogenic addiction and risks associated with prescribed opioids are higher than previously believed, when as early as 2001 the rate of hospitalization due to heroin was overtaken by non-heroin opioids [5,8]. Other factors contributing to this growth include more attempts to increase patient satisfaction scores, as well as strong marketing of opioids by the pharmaceutical industry [9-11].

Due to this increase in opioid use, there has also been an increase in opioid overdoses and opioid associated deaths, as mentioned above [6]. Research proposes that certain contributing factors such as low socioeconomic status, being of male gender, concurrent use of multiple medications, recent incarceration, homelessness and mental health conditions increase the likelihood of opioid overdose, from both prescription and illicit opioids [12-14]. This trend has not been limited to Australia but is being observed around the world with the United States (US) stating it is in an "opioid epidemic" [15].

With this increased number of accidental opioid-related deaths around the world, there has been a global shift to increase access to take-home naloxone for administration by patients and bystanders. Naloxone is a "rescue drug" that was approved for use by the US Food and Drug Administration (FDA) in 1971 for administration by emergency medical providers [16]. Naloxone is a mu-opioid receptor antagonist with the ability to reverse the effects of opioids on the central nervous system and improve acute respiratory status $[17,18]$. It is deliverable via injection or intranasal routes, with similar efficacy [19]. Patient preference and ease of administration in a non-hospital setting lean toward intramuscular or intranasal use; however, intranasal forms are not readily available in some countries, including Australia [19]. In small doses, naloxone is also indicated for reducing constipation associated with chronic opioid use; however, this is not the focus of this review [20].

Many countries around the world, including various states in the US, the United Kingdom, Canada, Italy and Australia have made naloxone available without a prescription, in the hope that those at risk of an opioid overdose, or their family and friends (bystanders), can easily have access to this life-saving medication [21-24]. The down-scheduling of naloxone occurred in Australia in February 2016, shifting it to Schedule Three (Pharmacist Only) medicine to be accessed without a prescription [25]. Prior to this, naloxone was available as a "Prescription-Only" medicine, primarily used in emergency medical service and hospital settings [25]. In 2011, a program named "Implementing Expanded Naloxone Availability in the Australian Capital Territory" (IENAACT) was commenced, trialling an increase in naloxone availability and awareness in the Australian Capital Territory (ACT) community [26]. This program ascertained that training participants (mostly opioid users) allowed successful administration of naloxone in an overdose situation, and that participants felt "positive emotional impacts" [26]. There were also 96 individual submissions to the Therapeutic Goods Administration (TGA), all reiterating that making naloxone "over the counter" will remove a barrier to its access, and that it is safe and holds no potential for misuse or abuse [23]. The successful outcomes of the IENAACT trial, the submissions to the TGA and a recommendation from The Advisory Committee on Medicines Scheduling (ACMS), all aided in the final decision to down-schedule naloxone [25].

Although policy changes such as this should markedly increase access to naloxone and its use in the community, the level of uptake and outcomes of take-home naloxone have not been clear. Furthermore, it is unknown if any specific challenges have arisen, which may be influencing the actions or opinions of all parties involved in take-home naloxone supply and use. Hence, the aim of this scoping review was to explore the perceived factors influencing the use of take-home naloxone from the perspectives of different stakeholders. 


\section{Materials and Methods}

A scoping review methodology, following the Preferred Reporting Items for Systematic Reviews and Meta-Analyses (PRISMA) checklist, was adopted for this study in order to "describe in more detail the findings and range of research in particular areas of study, thereby providing a mechanism for summarizing and disseminating research findings to policy makers, practitioners and consumers" [27,28].

\subsection{Search Strategy}

The literature was searched and retrieved systematically from three databases: PubMed, EMBASE and MEDLINE on the 30 March 2017, in accordance with PRISMA guidelines. A variation of text words, key words and MeSH terms/subject headings were used: "*naloxone" AND "opioid analgesic"

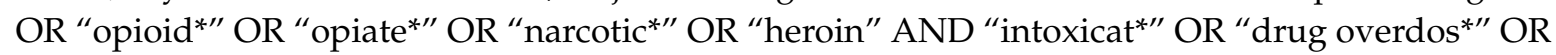
"overdos". See Table A1 for an example of the search strategies.

\subsection{Study Selection}

The study inclusion criteria included primary research articles published in the last ten years (2007-2017), because the injectable product for this purpose was introduced recently on the market, in peer reviewed journals focusing on stakeholders' perceptions of factors affecting the uptake of take-home naloxone and its use anywhere around the world. Exclusion criteria were: language (not English) and studies focusing on pharmacologic mechanisms of actions, side effect profiles, dosage forms and naloxone in a non-take-home context, such as its use within a hospital. Articles that were not primary research and were excluded from selection included editorials, conference abstracts, notes, letters to the editor, reviews, case reports and supplements.

In the first phase of screening, duplicates, studies which were not primary research and those that did not meet our inclusion criteria based on the title, were removed. In the second phase of screening, abstracts were reviewed. Studies not in English were also excluded prior to the full text screening phase. Next, full texts were screened to focus on topics which met our inclusion criteria. Lastly, studies not identified by the database searches were sought by hand by searching the grey literature and the bibliographies of publications and were added to the pool of literature where relevant.

\subsection{Assessment of Study Quality}

The quality of included studies was assessed using the Joanna Briggs Institute (JBI) Critical Appraisal Checklists. The JBI Critical Appraisal Checklists utilized were specific to the various methodologies employed within the included studies. Within the checklists, each item was scored 0 , 0.5 or 1 , with higher scores indicating greater quality. As the checklists for various study methodologies contain between 8 and 13 items, scores were normalized to give a final score out of 100 to allow comparison across study types. Studies scoring above 75 were considered high quality, those scoring between $50-75$ were considered medium quality and those scoring below 50 were considered low quality.

\subsection{Data Extraction and Analysis}

The following data were extracted and collated from the articles predominantly by one of the researchers (TH): country of study, data collection method, sample size, brief method and intervention details, main outcomes and funding source. The studies were then organized based on the sample population or perspectives. Studies were further analysed based on their study design, major findings and themes. Themes were then discussed and clarified (by TH, JP and BC) until a consensus was reached. 


\section{Results}

The search strategy generated 428 articles from MEDLINE, 474 from EMBASE and 581 from PubMed, yielding 1483 in total. After removal of duplicates, 978 were screened and through various phases of elimination 51 studies were included for analysis as shown in Figure 1. The mean study quality score was 76 (standard deviation \pm 17 ) indicating most were of medium to high quality.

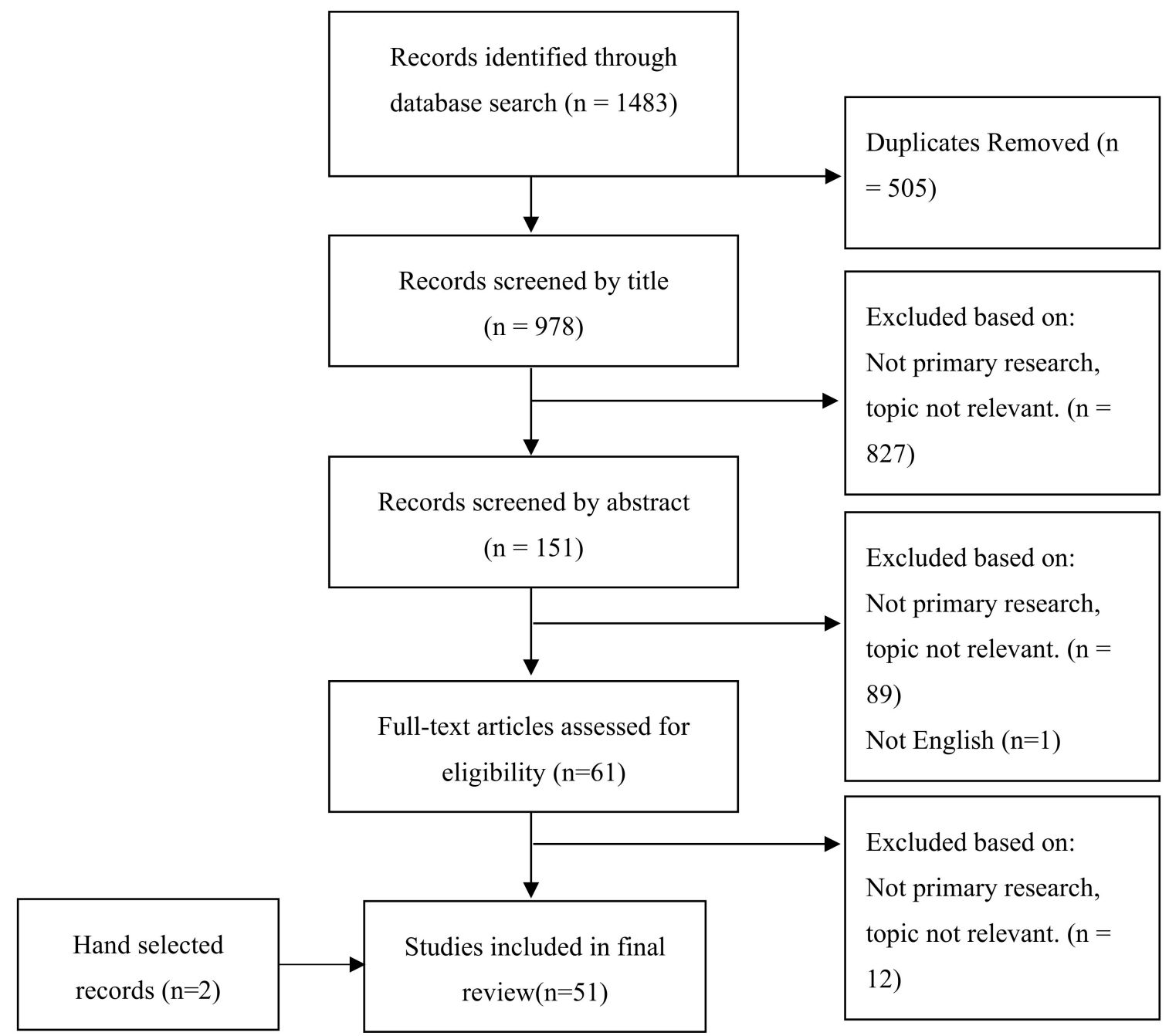

Figure 1. Preferred Reporting Items for Systematic Reviews and Meta-Analyses (PRISMA) Flow Diagram of Study Selection.

Studies were categorized into two groups pertaining to the perspective that each was based on. Thirty-six studies were found relating to the perspectives of patients likely to receive naloxone, and bystanders, who were members of the public who did not have an opioid addiction themselves, but were mostly friends and family of people who were at risk of opioid overdose. Of these, $64 \%$ were high quality, $25 \%$ medium quality and $11 \%$ low quality studies.

Fifteen studies were found exploring healthcare professionals' perspectives, including various medical professionals, staff from prisons, needle exchange facilities and homeless shelter programs. In total, thirty-eight studies were conducted in the United States, seven in the United Kingdom, four in Canada, one in Norway and one in Australia. Of these, 60\% were high quality and 40\% were medium quality. 


\subsection{Patients' and Bystanders' Perspectives}

The most prominent finding (24/36) within the patients' and bystanders' perspectives category was the positive outcomes resulting from access and training to take-home naloxone in terms of knowledge, confidence and rate of opioid reversals [29-52]. Four studies identified several facilitators including: the fact that naloxone is a life-saving measure; that it has the ability to empower people and potentially decrease drug use and that training was novel and interesting [53-56]. Some barriers identified included: the delivery of information from healthcare professionals as "professionally led health promotion initiatives appeared to lack credibility amongst the target population", that administration can be challenging due to the potential need to titrate doses and the use of a needle in all routes of use other than intranasal administration. There was also fear of the unpleasant withdrawal symptoms that naloxone almost immediately precipitates, colloquially known as "dopesickness". Furthermore, there was apprehension toward calling emergency services due to the fear of police interaction and the potential for incarceration [53-56].

In regard to the types of studies, pre-post training assessments was the most common (16/36) [30,32-35,37,40,41,43-45,48,49,52,57,58], followed by 11 cross-sectional surveys/questionnaires $[29,31,36,39,42,50,59-63]$ and eight interviews $[38,46,53-56,58,64]$. There was one randomized control trial in the entire sample [51].

Needle exchange programs, homeless shelters, injecting drug user (IDU)-based surveys and other similar facilities were the main setting or source of recruitment for the studies (30/36) [29-36,38-50,52-56,60,62-64]. One study, conducted in an emergency department setting, which offered take-home naloxone to those deemed eligible, found that the majority of patients believed it was a "good idea" and that there is potential for emergency department based distribution to increase access to those most vulnerable to overdose [61].

\subsection{Healthcare Professionals' Perspectives}

Identification and recognition of key facilitators and barriers to increasing naloxone access were the predominant themes of this category (11/15), unlike the patient and bystander results [65-75]. Seven of these studies were in the form of discussion groups and interviews, and the remaining four were surveys. Some of the facilitating aspects recognised were interventions that were "real-world" driven, provided education and training, had available resources and current involvement or awareness of other harm-reduction programs (such as opioid substitution therapy). Some of the perceived barriers included: financial and other logistical difficulties such as lack of staff and time to appropriately train and educate patients and bystanders, regulations and legalities and lack of education and training. Examples of specific patient-related barriers included concerns regarding offending patients who had not previously experienced an overdose in response to the offer of take-home naloxone as well as a stigma. Stigma is multifaceted, including patients being discriminated against by peers for having this medication, and is related to some healthcare professionals' expressing lowered motivation and interest toward helping people who are using opioids. Two pre-post evaluation studies showed positive outcomes from healthcare professionals receiving additional training for the use of take-home naloxone [76,77].

Two studies were associated with a needle exchange clinic or safe injecting room types of settings [74,77]. Four studies were conducted from a pharmacy perspective, with two of them identifying similar facilitators and barriers as mentioned above $[65,67]$ and two showing mostly positive attitudes of pharmacists toward take-home naloxone but also highlighting a lack in knowledge [71,75]. Emergency care provider opinions were the focus of two studies, one concluding a predominantly negative attitude toward take-home naloxone with the opinion that it would not decrease death rates [73]. The second identified several facilitators and barriers as mentioned above [67]. 


\section{Discussion}

This review has explored the literature available in regard to factors influencing the use of take-home naloxone from the perspectives of patients/bystanders and healthcare professionals. From the perspectives of patients and bystanders, the findings in the literature depicted positive responses from the increased access to take-home naloxone [29-52]. A sense of empowerment increase in confidence and ability to recognize overdose symptoms were just some of the encouraging conclusions that these studies made. In conjunction with these findings, it was clear that naloxone administrations were successful in reducing opioid-related overdose deaths, which is ultimately the goal of all harm minimization interventions [30,32-35,38,39,41-48,50,52].

In 1985, the Australian Government adopted harm minimization as a national framework in an attempt to address the range of drug and alcohol issues in society [78]. Whilst it is clear that take-home naloxone has support from its potential users in the community as a harm minimization project, healthcare professionals have expressed concerns about its uptake, as it was perceived to encourage high-risk opioid use [66,68-70,72-75]. Similar concerns were, and still are, expressed in regard to needle exchange and distribution programs [79]. Needle exchange programs have been implemented in Australia since the mid-1980s under the harm minimization framework mentioned above [79]. Although there has been no evidence that take-home naloxone or syringe exchange programs increased drug use, this stigma still remains [80-82]. In fact, studies have shown that naloxone has the potential to decrease drug use, as having access to naloxone motivated and empowered patients to be more health conscious $[33,45]$.

To highlight the stigma associated with naloxone, a comparison can be drawn with adrenaline for anaphylaxis. Both naloxone and adrenaline are patient administered rescue medications that save lives; however, the introduction of adrenaline induced no resistance from the community compared to other harm minimization programs such as syringe exchange or methadone [83]. A systematic review around healthcare professionals' perspectives showed that they expressed "lowered regard, less motivation and feelings of dissatisfaction" toward patients with substance use disorders, consolidating this notion of stigma [84]. As mentioned, naloxone is just as much a life-saving medication as adrenaline, and a healthcare professional's decision to withhold it from patients based on this stigma is a violation of all principles of professional ethics in healthcare $[85,86]$. Codes of ethics state that, despite a conscientious objection to the supply or prescribing of a medical product, healthcare professionals have an obligation to place the best interests of the patient above all else and, at the very least, maintain continuity of care to all patients $[85,86]$.

It is also known that illicit use of opioid medication, prescription or not, is not the only cause of opioid overdose [87]. Chronic pain patients are also at risk of opioid overdoses due to pharmacokinetic changes with age or confusion about dosing or instructions of use [88]. Despite this, current studies are strongly focused on injecting drug users and patients involved with needle exchange programs, homeless shelters and similar facilities. This disproportionate focus may be due to the fact that talking to non-illicit opioid users about take-home naloxone was identified as a barrier by many healthcare professionals' due to the fear of offending them $[67,68]$. A way to mitigate this risk would be to educate healthcare professionals on how to identify "high risk" chronic opioid using patients for the potential of opioid overdose and provide all of these patients with take-home naloxone [89,90].

Many healthcare professionals also emphasized the lack of education and training on take-home naloxone in this review [67-73,75]. Although codes of ethics in healthcare also state that healthcare professionals are bound by an obligation to be life-long learners, it is clear that patients are being adversely affected by healthcare professionals' lack of knowledge $[85,86]$. Two studies showed that training lasting around an hour was sufficient to increase the knowledge of homeless shelter staff and other healthcare providers [76,77]. All healthcare professionals who prescribe opioids or care for patients at risk of opioid overdose should be provided with training on take-home naloxone.

Barriers identified in this review have all been encountered previously by other harm prevention strategies such as the methadone substitution therapy. Methadone programs were introduced in 
Australia in the 1970s and over time have slowly overcome barriers associated with training, education and stigma, similar to those identified by the professionals' perspectives category in this review $[91,92]$. A systematic review about stigma among healthcare professionals towards patients with substance use disorder stressed the importance of training of healthcare professionals "in order to extend the knowledge, skills and self-efficacy of professionals working with patients with substance use disorders" [84]. Alongside this training, two factors were identified by McArther 1999 that assisted methadone in gaining traction in communities and overcoming these barriers including the high demand for it from drug users themselves, and the eventual realization that it played a role in reducing crime rates and reducing HIV/AIDs transmission [92]. This last point indicates that with time, naloxone could gain community awareness, proving its worth and benefits in a take-home setting, as was the case for methadone. In fact, an article in 2007 by Beletsky et al. supported this notion and concluded that physicians with more experience and awareness of patients with substance use disorders were more inclined to respond positively to take-home naloxone prescriptions [93].

Emergency care providers were highlighted in this review as they hold expertise in opioid-related overdoses and take-home naloxone has the potential to impact the nature of their interactions with overdosing patients $[67,73]$. However, emergency providers were found to hold negative views towards patients with substance use disorders, with one study showing that more than half viewed take-home naloxone training as an ineffective strategy to reduce opioid-related deaths [73]. Addressing emergency providers' concerns and obtaining their support is crucial for successful uptake of take-home naloxone. In addition to the large number of patients that present to the emergency department (ED) that are at risk of an opioid overdose, evidence also shows that patients are likely to stay on the medications prescribed for them in hospital [94]. With this trend in mind, it is imperative that emergency physicians are the focus point for further education about take-home naloxone, in order to increase the dissemination of this life-saving medication into the community. Once the initial uptake has been established, it is assumed that other healthcare providers such as general practitioners and pharmacists are likely to follow this pattern of distribution.

The perspective of the pharmacist was also explored in this review $[66,68,72,76]$. As take-home naloxone no longer requires a prescription in many countries, pharmacists are becoming increasingly involved in its distribution. Pharmacists are arguably the most accessible healthcare professionals and may be the first point of contact patients have with the healthcare system [72]. Two studies communicated policy regulations as a large barrier to take-home naloxone from a pharmacist's perspective. Regulatory issues are particularly prevalent in the US, where legislation differs between states, causing confusion for all parties involved, in relation to the varying degrees of access to take-home naloxone $[95,96]$. Furthermore, although pharmacists were found to express positive attitudes towards harm-reduction services, very few stocked naloxone and the majority lacked confidence in their ability to educate patients on naloxone use [72]. Another study noted that pharmacists were supportive of take-home naloxone, but were unaware of the high prevalence of opioid overdose [76]. This limited cognizance regarding take-home naloxone and the opioid burden in general is reflected in the lack of uptake of this medicine in the community. The paucity of information surrounding the role of pharmacists and emergency care professionals, two key stakeholders in the future of take-home naloxone, is an area necessitating further research.

Limitations: The current findings need to be considered in light of several limitations. The review was limited to articles in English; however, there was only one study identified that was not in English, thus it is unlikely that this restriction greatly impacted the results [96]. Second, the majority of the studies included were from the US, limiting the applicability or generalizability of these results to a worldwide setting. Third, only three databases were used for the systematic search strategy; however, this included two of the largest, most comprehensive databases in this area of research. Future researchers could conduct a similar search in databases such as Cumulative Index to Nursing and Allied Health Literature (CINAHL) and International Pharmaceutical Abstracts (IPAs), in order to 
gain additional literature from a broader range of medical professions. Despite this, we believe that this search was sufficient to support our findings and that all key themes and perspectives were identified.

\section{Conclusions}

Findings of this study indicated that patients and bystanders who may use take-home naloxone were eager and have positive attitudes towards its use; however, there remain some barriers from a healthcare professional's perspective. In particular, it was found that stigma around drug use negatively affects the implementation/uptake of take-home naloxone, as some healthcare professionals appear to view these patients. Future research should be aimed at exploring how to gain stronger support from emergency care professionals who are experienced with opioid overdoses and the potential impact of take-home naloxone on the community. The obstacles identified in this study, regarding the implementation of take-home naloxone, were not new concepts. With time, allowing for the efficacy of naloxone to be proven, and with an increase in education and training for all parties involved, more patients at risk of opioid overdose should see access to this life-saving medication. In addition, the impact of take-home naloxone should be explored more extensively in settings outside of the US.

Author Contributions: All authors contributed to the conceptualization of this manuscript. T.J.H. conducted most of the literature extraction and J.P., J.J., M.S. and B.B.C. assisted with the write up of analysis and discussion. All authors have read and agreed to the published version of the manuscript.

Funding: This research received no external funding.

Acknowledgments: This study was conducted as part of Taylor Holland's Honours degree requirements and only received kind support from the University of Sydney.

Conflicts of Interest: The authors declare no conflict of interest.

\section{Appendix A}

Table A1. Example search strategy from medline.

\begin{tabular}{ccc}
\hline$\#$ & Search Statement & Results \\
\hline 1 & * naloxone/ & 20,831 \\
2 & opiate/ & 79,558 \\
3 & narcotic agent/ & 6672 \\
4 & heroin.tw. & 16,875 \\
5 & 2 or 3 or 4 & 107,585 \\
6 & intoxication/ & 216,931 \\
7 & drug overdose/ & 20,940 \\
8 & 6 or 7 & 230,238 \\
9 & 1 and 5 and 8 & 544 \\
10 & limit 9 to human & 474 \\
\hline
\end{tabular}

* The asterix in this context is what is known as a "wildcard" and represents any group of characters, including no character. 
Table A2. Summary of Included Studies from Patients' and Bystanders' Perspectives.

\begin{tabular}{|c|c|c|c|c|c|c|}
\hline \multicolumn{7}{|c|}{ Patients' and Bystanders' Perspectives } \\
\hline Author, Title and Journal & Country & Data Collection Method & Participants & Interventions/Study Details & Outcomes & $\begin{array}{l}\text { Funding/ } \\
\text { Sponsorship }\end{array}$ \\
\hline $\begin{array}{l}\text { Bachhuber et al., } \\
2015 \\
\text { Messaging to increase public } \\
\text { support for naloxone } \\
\text { distribution policies in the } \\
\text { United States: results from a } \\
\text { randomized survey } \\
\text { experiment. PLOS One [58] }\end{array}$ & US & $\begin{array}{l}\text { Cross-sectional survey } \\
\text { post-randomized exposure } \\
\text { to intervention }\end{array}$ & $\begin{array}{c}\mathrm{N}=267 \text { Control }(\mathrm{C}) \\
\mathrm{N}=260 \text { Factual }(\mathrm{F}) \\
\mathrm{N}=266 \text { Factual }+ \\
\text { Refutation }(\mathrm{FR}) \\
\mathrm{N}=264 \\
\text { Sympathetic narrative (SN) } \\
\mathrm{N}=276 \\
\text { Sympathetic + Factual (SF) } \\
50.1 \% \text { women, } 73.5 \% \text { white, } \\
22 \% 55-64 \text { years old }\end{array}$ & $\begin{array}{l}\text { Random selection of US } \\
\text { households from GfK survey } \\
\text { research panel were exposed to } \\
\text { three different types of } \\
\text { persuasive messages for } \\
\text { support of naloxone alone or in } \\
\text { combination (or compared too } \\
\text { no exposure) and support for } \\
\text { naloxone distribution polices } \\
\text { was assessed }\end{array}$ & $\begin{array}{l}\text { - Support from C was strongest for } \\
\text { training first responders }(63.2 \%) \text { and } \\
\text { passing laws to protect people if they } \\
\text { call for medical help ( } 52.4 \%) \\
-77.4 \% \text { of } \mathrm{F} \text { group supported training } \\
\text { first responders and } 80.5 \% \text { of } \mathrm{SN} \text { group } \\
-58 \% \text { of } \mathrm{F} \text { and } 55.6 \% \text { of SN groups } \\
\text { supported passing laws to protect those } \\
\text { calling for medical help } \\
-45.5 \% \text { of } \mathrm{F} \text { group supported providing } \\
\text { naloxone to family members and } 40.1 \% \\
\text { of the SN group } \\
-73.7 \% \text { of } \mathrm{F}, 77 \% \text { of } \mathrm{SN} \text { believed naloxone } \\
\text { would save lives (60\% of control) } \\
\text { - SF group had high support for all } \\
\text { outcomes }\end{array}$ & $\begin{array}{l}\text { American } \\
\text { International } \\
\text { Group Inc }\end{array}$ \\
\hline $\begin{array}{c}\text { Bagley et al., } \\
2015 \\
\text { Overdose education and } \\
\text { naloxone rescue kits for family } \\
\text { members of individuals who } \\
\text { use opioids: characteristics, } \\
\text { motivations, and naloxone use. } \\
\text { Substance Abuse [29] }\end{array}$ & US & Cross-sectional survey & $\begin{array}{c}\mathrm{N}=126 \text { family members of } \\
\text { opioid users }\end{array}$ & $\begin{array}{c}\text { A convenience sample of } \\
\text { community support group } \\
\text { attendees was surveyed } \\
\text { The community group offered } \\
\text { naloxone training lasting } 20 \\
\text { min. } \\
\text { Compared those who received } \\
\text { overdose education and } \\
\text { naloxone to those who did not. }\end{array}$ & $\begin{array}{l}\text {-Those who received training were more } \\
\text { likely to be a parent }(91 \% \text { versus } 65 \%) \\
\text { and have daily contact with opioid user } \\
\text { ( } 54 \% \text { versus } 33 \% \text { ) } \\
-60 \% \text { felt encouraged from education at } \\
\text { meetings, } 72 \% \text { wanted naloxone in the } \\
\text { house } \\
\text { - Trainees endorsed "greater sense of } \\
\text { security" and "improved confidence" } \\
\text { - Of the } 27 \text { untrained subjects, } 2 \text { reported } \\
\text { it was not necessary for training and } 2 \\
\text { reported not living with the user }\end{array}$ & $\begin{array}{l}\text { National } \\
\text { Institute on } \\
\text { Drug Abuse }\end{array}$ \\
\hline $\begin{array}{l}\text { Barocas et al., } \\
2015 \\
\text { High uptake of naloxone-based } \\
\text { overdose prevention training } \\
\text { among previously incarcerated } \\
\text { syringe-exchange program } \\
\text { participants. Drug and Alcohol } \\
\text { Dependence [59] }\end{array}$ & US & Cross-sectional survey & $\begin{array}{c}\mathrm{N}=309 \text { people who use } \\
\text { drugs with no history of } \\
\text { incarceration } \\
\mathrm{N}=234 \text { people who use } \\
\text { drugs with history of } \\
\text { incarceration } \\
\text { Mean age } 31.6,79 \% \text { white, } \\
69 \% \text { male }\end{array}$ & $\begin{array}{l}\text { Participants were injecting } \\
\text { drug users from Lifepoint } \\
\text { Needle Exchange program }\end{array}$ & $\begin{array}{l}\text { - 198/234 ( } 85 \%) \text { had observed and } 96 / 234 \\
(41 \%) \text { had experienced an overdose } \\
\text { compared to 174/309 (56\%) and } 70 / 309 \\
\text { (23\%) of the group who had no history } \\
\text { of incarceration } \\
-162 / 234(69 \%) \text { of the history of } \\
\text { incarceration group had been trained to } \\
\text { administer naloxone compared to } \\
175 / 309(57 \%) \text { of the non-incarcerated } \\
\text { groupand } 98 / 234(46 \%) \text { had } \\
\text { administered naloxone compared to } \\
85 / 309(37 \%)\end{array}$ & $\begin{array}{l}\text { Clinical and } \\
\text { Translational } \\
\text { Science Award }\end{array}$ \\
\hline
\end{tabular}


Table A2. Cont.

\begin{tabular}{|c|c|c|c|c|c|c|}
\hline \multicolumn{7}{|c|}{ Patients' and Bystanders' Perspectives } \\
\hline Author, Title and Journal & Country & Data Collection Method & Participants & Interventions/Study Details & Outcomes & $\begin{array}{l}\text { Funding/ } \\
\text { Sponsorship }\end{array}$ \\
\hline $\begin{array}{c}\text { Bennett et al., } \\
2011 \\
\text { Characteristics of an overdose } \\
\text { prevention, response, and } \\
\text { naloxone distribution program } \\
\text { in Pittsburgh and Allegheny } \\
\text { County, Pennsylvania. Journal } \\
\text { of Urban Health: Bulletin of } \\
\text { the New York Academy of } \\
\text { Medicine [30] }\end{array}$ & US & $\begin{array}{c}\text { Pre-post survey } \\
\mathrm{T}_{0} \text { : Baseline before training } \\
\mathrm{T}_{\mathrm{R}} \text { : Returning for refill }\end{array}$ & $\begin{array}{c}\mathrm{N}=426 \text { people who use } \\
\text { drugs trained in naloxone } \\
\text { use }\end{array}$ & $\begin{array}{l}\text { Training }(25 \mathrm{~min}) \text { on } \\
\text { prevention, identification and } \\
\text { response to an overdose and } \\
\text { how to administer naloxone, } \\
\text { rescue breathing and calling } \\
911 \\
\text { Volunteer physicians } \\
\text { prescribed naloxone }\end{array}$ & $\begin{array}{l}\mathrm{T}_{0}: 170 / 426(40 \%) \text { had overdosed in their } \\
\text { life } \\
\text { - EMS called } 33 \% \text { of times } \\
\mathrm{T}_{\mathrm{R}}: 89 / 141(63 \%) \text { reported using } \\
\text { naloxone } \\
\text { - EMS called } 10 \% \text { of times } \\
-85 / 89(96 \%) \text { reported positive outcomes } \\
\text { after naloxone administration } \\
-61 \% \text { performed rescue breathing they } \\
\text { were taught in training }\end{array}$ & $\begin{array}{c}\text { National } \\
\text { Institute of Drug } \\
\text { Abuse }\end{array}$ \\
\hline $\begin{array}{c}\text { Bird et al., } \\
2016 \\
\text { Effectiveness of Scotland's } \\
\text { National Naloxone Programme } \\
\text { for reducing opioid-related } \\
\text { deaths: a before (2006-10) } \\
\text { versus after (2011-13) } \\
\text { comparison. } \\
\text { Addiction [31] }\end{array}$ & UK & $\begin{array}{l}\text { Cross-sectional record } \\
\text { analysis; two time points } \\
\text { investigated }\end{array}$ & $\begin{array}{l}\mathrm{N}=193 \text { opioid-related } \\
\text { deaths in } 2006-10 \text { within } 4 \\
\text { weeks of prison release } \\
\mathrm{N}=76 \text { opioid-related } \\
\text { deaths in } 2011-12 \text { within } 4 \\
\text { weeks of prison release }\end{array}$ & $\begin{array}{l}\text { The national naloxone } \\
\text { programme provides free } \\
\text { naloxone and training to all } \\
\text { those at risk of opioid } \\
\text { overdose, including prisoners } \\
\text { at liberation } \\
\text { National Records of Scotland } \\
\text { release official statistics on the } \\
\text { number of drug-related deaths }\end{array}$ & $\begin{array}{l}\text { - Reduction in prison release } \\
\text { opioid-related death by 3.5\% (9.8\%, } \\
\text { 193/1970, versus 6.3\%,76/1212) } \\
\text { - Distribution of 11,898 naloxone kits } \\
\text { assumed to have prevented } 42 \text { prison } \\
\text { release opioid-related deaths }\end{array}$ & $\begin{array}{l}\text { Medical } \\
\text { Research } \\
\text { Council }\end{array}$ \\
\hline $\begin{array}{l}\text { Delaney et al., } \\
2016 \\
\text { Coprescribing naloxone for } \\
\text { patients on chronic opioid } \\
\text { therapy: lessons learned from a } \\
\text { patient-safety initiative in } \\
\text { primary care training sites. } \\
\text { Journal of Opioid Management } \\
\text { [56] }\end{array}$ & US & $\begin{array}{l}\text { Pre-post questionnaire: } \\
\mathrm{T}_{0} \text { : Baseline naloxone } \\
\text { prescription through } \\
\text { medical records } \\
\mathrm{T}_{\mathrm{P}} \text { : Obtaining a } \\
\text { prescription }\end{array}$ & $\begin{array}{c}\mathrm{N}=204 \text { people who use } \\
\text { opioids }\end{array}$ & $\begin{array}{l}\text { Resident physicians initiate } \\
\text { patient selection regarding } \\
\text { naloxone prescription } \\
\text { Participants were taught about } \\
\text { naloxone, first by the resident } \\
\text { but part way through the study } \\
\text { changed to the pharmacist }\end{array}$ & $\begin{array}{l}\mathrm{T}_{0} \text { : No co-prescriptions of naloxone } \\
\text { written } \\
\mathrm{T}_{\mathrm{P}}: 53 / 204(25 \%) \text { of patients were written } \\
\text { prescriptions } \\
\text { - Patients who accepted the prescription } \\
\text { often had been exposed to an overdose }\end{array}$ & None stated \\
\hline $\begin{array}{c}\text { Doe-simkins et al., } \\
2014 \\
\text { Overdose rescues by trained } \\
\text { and untrained participants and } \\
\text { change in opioid use among } \\
\text { substance-using participants in } \\
\text { overdose education and } \\
\text { naloxone distribution } \\
\text { programs: a retrospective } \\
\text { cohort study. BMC Public } \\
\text { Health [32] }\end{array}$ & US & $\begin{array}{c}\text { Retrospective cohort study. } \\
\text { post-questionnaire: } \\
\mathrm{T}_{0} \text { : Baseline demographics } \\
\mathrm{T}_{\mathrm{R}}: \text { Returning for refill }\end{array}$ & $\begin{array}{c}\mathrm{N}=295 \text { trained } \\
\mathrm{N}=78 \text { untrained people } \\
\text { who use drugs }\end{array}$ & $\begin{array}{l}\text { Training }(5-60 \text { min) included } \\
\text { overdose prevention, assessing } \\
\text { for overdose, seeking help, } \\
\text { rescue breathing, naloxone } \\
\text { administration, } \\
\text { post-administration support } \\
\text { Using data from Massachusetts } \\
\text { Opioid Overdose Prevention } \\
\text { Pilot Program (restricted from } \\
\text { September } 18,2006 \text { to } \\
\text { December } 312010)\end{array}$ & $\begin{array}{l}T_{R}: \text { No significant differences in the } \\
\text { overdose event if the rescuer was } \\
\text { previously trained or not } \\
\text { - Naloxone was used successfully } \\
295 / 303(97 \%)\end{array}$ & $\begin{array}{c}\text { Centers for } \\
\text { Disease Control } \\
\text { and Prevention }\end{array}$ \\
\hline
\end{tabular}


Table A2. Cont.

\begin{tabular}{|c|c|c|c|c|c|c|}
\hline \multicolumn{7}{|c|}{ Patients' and Bystanders' Perspectives } \\
\hline Author, Title and Journal & Country & Data Collection Method & Participants & Interventions/Study Details & Outcomes & $\begin{array}{c}\text { Funding/ } \\
\text { Sponsorship }\end{array}$ \\
\hline $\begin{array}{c}\text { Dong et al., } \\
\text { (2012) } \\
\text { Community-based naloxone: a } \\
\text { Canadian pilot program. } \\
\text { Canadian Journal of Addiction } \\
\text { Medicine [33] }\end{array}$ & Canada & $\begin{array}{c}\text { Pre-post questionnaire: } \\
\mathrm{T}_{0}: \text { Baseline before training } \\
\mathrm{T}_{\mathrm{a}}: \text { Immediately after } \\
\text { training } \\
\mathrm{T}_{1}: 1 \text { year after training }\end{array}$ & $\begin{array}{l}\mathrm{N}=50 \text { clients, Streetworks } \\
\text { needle exchange program }\end{array}$ & $\begin{array}{l}\text { Individual training ( } 30-40 \mathrm{~min}) \\
\text { focused on harm reduction, } \\
\text { including overdose prevention, } \\
\text { recognition of overdose, } \\
\text { provision of respiration, } \\
\text { naloxone education and IM } \\
\text { administration and calling } \\
\text { EMS. Optional CPR was } \\
\text { offered }\end{array}$ & $\begin{array}{l}\mathrm{T}_{0}: 39 / 50(78 \%) \text { had experienced an } \\
\text { overdose and } 46 / 50(92 \%) \text { had witnessed } \\
\text { an overdose } \\
\mathrm{T}_{\mathrm{a}}: \text { Naloxone use reported } 9 \text { times, all } \\
\text { successful outcomes } \\
-9 / 9 \text { used a clean syringe } \\
-8 / 9 \text { alcohol swabbed the skin before } \\
\text { injection } \\
-1 / 9 \text { EMS contacted } \\
-1 / 9 \text { participant felt unsure what to do } \\
\mathrm{T}_{1}: 15 / 15 \text { found training worthwhile } \\
-11 / 15 \text { reported decrease in drug use }\end{array}$ & Health Canada \\
\hline $\begin{array}{c}\text { Enteen et al., } \\
2010 \\
\text { Overdose prevention and } \\
\text { naloxone prescription for } \\
\text { opioid users in San Francisco. } \\
\text { Journal of Urban Health [34] }\end{array}$ & US & $\begin{array}{c}\text { Pre-post questionnaire: } \\
\mathrm{T}_{\mathrm{a}} \text { : immediately after } \\
\text { training } \\
\text { Interviews or questionnaire: } \\
\mathrm{T}_{\mathrm{r}} \text { : receiving naloxone } \\
\text { refills }\end{array}$ & $\begin{array}{l}\mathrm{N}=470 \text { people who use } \\
\text { drugs received a naloxone } \\
\text { refill }\end{array}$ & $\begin{array}{l}\text { Training was } 10-30 \mathrm{~min} \\
\text { focusing on overdose } \\
\text { identification, revival, calling } \\
\text { EMS and administering } \\
\text { naloxone } \\
\text { Physicians prescribed and } \\
\text { dispensed naloxone }\end{array}$ & $\begin{array}{l}\mathrm{T}_{\mathrm{r}}: 215 / 470 \text { naloxone supplies were used } \\
\text { in overdose and } 311 / 470 \text { were reported } \\
\text { lost } \\
-357 / 399 \text { overdose events were } \\
\text { successful } \\
-116 / 399 \text { reported calling EMS and } \\
123 / 399 \text { reported using an additional } \\
\text { strategy they were taught }\end{array}$ & None stated \\
\hline $\begin{array}{c}\text { Galea et al., } \\
2006 \\
\text { Provision of naloxone to } \\
\text { injection drug users as an } \\
\text { overdose prevention strategy: } \\
\text { early evidence from a pilot } \\
\text { study in New York City. } \\
\text { Addictive Behaviors [22] }\end{array}$ & US & $\begin{array}{c}\text { Pre-post questionnaire: } \\
\mathrm{T}_{0}: \text { Baseline before training } \\
\mathrm{T}_{3 \mathrm{~m}}: 3 \text { months after } \\
\text { training }\end{array}$ & $\begin{array}{c}\mathrm{N}=25 \text { needle exchange } \\
\text { program clients }\end{array}$ & $\begin{array}{l}\text { Training focused on assessing } \\
\text { overdose victim, calling EMS, } \\
\text { positioning and rescue } \\
\text { breathing and administration } \\
\text { of up to two doses of naloxone } \\
\text { IM over one hour Conducted } \\
\text { in small groups or individually } \\
\text { Physician met with participants } \\
\text { to distribute naloxone }\end{array}$ & $\begin{array}{l}\mathrm{T}_{0}: 13 / 25 \text { experienced and } 19 / 25 \text { had } \\
\text { witnessed an overdose } \\
-11 / 19(58 \%) \text { called EMS } \\
\mathrm{T}_{3 \mathrm{~m}}: 9 / 11(82 \%) \text { called EMS } \\
-10 / 17 \text { naloxone administrations for } \\
\text { overdose, all } 10 \text { successful } \\
-15 / 20(75 \%) \text { felt comfortable using } \\
\text { naloxone } \\
\text { - Increase in appropriate response } \\
\text { techniques (placing in rescue position } \\
\text { from } 5.3 \% \text { to } 18.3 \% \text { ) }\end{array}$ & $\begin{array}{l}\text { Tides } \\
\text { Foundation }\end{array}$ \\
\hline $\begin{array}{c}\text { Green et al., } \\
2008 \\
\text { Distinguishing signs of opioid } \\
\text { overdose and indication for } \\
\text { naloxone: an evaluation of six } \\
\text { overdose training and } \\
\text { naloxone distribution } \\
\text { programs in the United States. } \\
\text { Addiction [35] }\end{array}$ & US & Cross-sectional survey & $\begin{array}{c}\mathrm{N}=30 \text { trained } \\
\mathrm{N}=32 \text { untrained people } \\
\text { who have or currently use } \\
\text { drugs } \\
72.6 \% \text { male, } 45.8 \% \\
\text { reporting previous } \\
\text { overdose }\end{array}$ & $\begin{array}{c}\text { Staff from six overdose training } \\
\text { and naloxone distribution sites } \\
\text { with similar training programs } \\
\text { agreed to participate } \\
\text { Staff from each site selected } 5 \\
\text { previously trained clients and } 5 \\
\text { untrained } \\
\text { Participants assessed } 16 \text { cases } \\
\text { about the nature of the } \\
\text { overdose }\end{array}$ & $\begin{array}{l}\text { - Knowledge of opioid overdose higher } \\
\text { among trained } 85.2 \% \text { versus } 68.3 \%(p< \\
0.005) \\
\text { - Similar indecision recognizing } \\
\text { non-overdose and non-opioid overdose } \\
(p=0.42) \\
\text { - Trained participants were more likely } \\
\text { to have responded to an OD } \\
\text { - Medical experts assigned } \\
\text { approximately } 10 \% \text { more codes to the } \\
\text { "don't know/unsure/not enough } \\
\text { information" category, compared to } \\
\text { trained respondents }\end{array}$ & $\begin{array}{l}\text { National } \\
\text { Institute of } \\
\text { Mental Health }\end{array}$ \\
\hline
\end{tabular}


Table A2. Cont.

\begin{tabular}{|c|c|c|c|c|c|c|}
\hline \multicolumn{7}{|c|}{ Patients' and Bystanders' Perspectives } \\
\hline Author, Title and Journal & Country & Data Collection Method & Participants & Interventions/Study Details & Outcomes & $\begin{array}{c}\text { Funding/ } \\
\text { Sponsorship }\end{array}$ \\
\hline $\begin{array}{c}\text { Jones et al., } \\
2014 \\
\text { Brief overdose education can } \\
\text { significantly increase accurate } \\
\text { recognition of opioid overdose } \\
\text { among heroin users. } \\
\text { International Journal of Drug } \\
\text { Policy [36] }\end{array}$ & US & $\begin{array}{c}\text { Pre-post assessment: } \\
\mathrm{T}_{0} \text { : Baseline before training } \\
\mathrm{T}_{\mathrm{a}}: \text { Immediately after } \\
\text { training }\end{array}$ & $\begin{array}{c}\mathrm{N}=44 \text { trained } \\
\mathrm{N}=40 \text { untrained people } \\
\text { who use heroin }\end{array}$ & $\begin{array}{l}\text { Semi-structured lecture } \\
\text { focused on risk factors, signs } \\
\text { and response for overdose } \\
\text { lasting } 13-18 \text { min } \\
\text { Control group did not receive } \\
\text { training }\end{array}$ & $\begin{array}{l}T_{0} \text { : Equivalent demographic breakdown } \\
\text { between the groups } \\
\text { - Assessment and confidence scores did } \\
\text { not differ between groups } \\
\mathrm{T}_{\mathrm{a}} \text { : Increase in trained groups increase } \\
\text { ability to identify opioid-overdose }(p< \\
0.05) \\
\text { - Trained increase confidence from } 7.9 \text { to } \\
9.4(p<0.05)\end{array}$ & $\begin{array}{l}\text { National } \\
\text { Institute on } \\
\text { Drug Abuse } \\
\text { grant }\end{array}$ \\
\hline $\begin{array}{c}\text { Kestler et al., } \\
2017 \\
\text { Factors associated with } \\
\text { participation in an emergency } \\
\text { department-based take-home } \\
\text { naloxone program for at-risk } \\
\text { opioid users. Annals of } \\
\text { Emergency Medicine [60] }\end{array}$ & Canada & Cross-sectional survey & $\begin{array}{l}\mathrm{N}=201 \text { emergency } \\
\text { department attendees } \\
37 \% \text { women, } 26 \% \\
\text { indigenous }\end{array}$ & $\begin{array}{l}\text { Participants recruited from } \\
\text { inner-city teaching and referral } \\
\text { center if they were over } 16 \text { and } \\
\text { high risk of opioid overdose } \\
\text { Five-minute training followed } \\
\text { by } 15-20 \text { min questionnaire, } \\
\text { and naloxone supply }\end{array}$ & $\begin{array}{l}\text { - 183/200 ( } 91.5 \%) \text { thought take-home } \\
\text { naloxone was a good idea and 168/200 } \\
(84 \%) \text { thought ED was a suitable location } \\
-137 / 201(68.2) \text { accepted naloxone kit } \\
\text { and brief training }\end{array}$ & None stated \\
\hline $\begin{array}{l}\text { Lankenau et al., } \\
\qquad 2013 \\
\text { Injection drug users trained by } \\
\text { overdose prevention programs: } \\
\text { responses to witnessed } \\
\text { overdoses. Journal of } \\
\text { Community Health [37] }\end{array}$ & US & Interviews & $\begin{array}{l}\mathrm{N}=30 \text { people who inject } \\
\text { drugs } \\
\begin{array}{c}\text { Majority male, non-white } \\
\text { and homeless }\end{array}\end{array}$ & $\begin{array}{l}\text { Participants from homeless } \\
\text { shelters, trained in naloxone } \\
\text { use were recruited } \\
\text { Series of open and closed } \\
\text { ended questions }\end{array}$ & $\begin{array}{l}\text { - Most participants first response was to } \\
\text { stimulate the victim } \\
-911 \text { was often called but with fear of } \\
\text { arrest } \\
\text { - CPR and rescue breathing was } \\
\text { administered } \\
\text { - Half injected naloxone, feeling capable } \\
\text { and with few reported difficulties }\end{array}$ & $\begin{array}{l}\text { National } \\
\text { Institutes of } \\
\text { Health }\end{array}$ \\
\hline $\begin{array}{c}\text { Leece et al., } \\
2013 \\
\text { Development and } \\
\text { implementation of an opioid } \\
\text { overdose prevention and } \\
\text { response program in Toronto, } \\
\text { Ontario. Canadian Journal of } \\
\text { Public Health [38] }\end{array}$ & Canada & Cross-sectional survey & $\begin{array}{c}\mathrm{N}=20 \text { people who use } \\
\text { opioids, trained in } \\
\text { naloxone use }\end{array}$ & $\begin{array}{l}\text { Participants recruited by word } \\
\text { of mouth and flyers } \\
\text { Training was } 20 \text { min exploring } \\
\text { prevention and response to } \\
\text { overdose, including calling } \\
\text { EMS, chest compression IM } \\
\text { naloxone administration and } \\
\text { post-overdose care }\end{array}$ & $\begin{array}{l}\mathrm{T}_{\mathrm{a}} \text { : Sense of empowerment after } \\
\text { completing the training } \\
-17 \text { naloxone administrations } \\
\text { post-training, all successful }\end{array}$ & None stated \\
\hline $\begin{array}{l}\text { Lott and Rhodes } \\
2016 \\
\text { Opioid overdose and naloxone } \\
\text { education in a substance use } \\
\text { disorder treatment program. } \\
\text { American Journal on } \\
\text { Addictions [39] }\end{array}$ & US & $\begin{array}{c}\text { Pre-post questionnaire: } \\
\mathrm{T}_{0} \text { : Baseline before training } \\
\mathrm{T}_{\mathrm{a}} \text { : Immediately after } \\
\text { training (training group } \\
\text { only) } \\
\mathrm{T}_{3 \mathrm{~m}}: \text { 3-month follow up }\end{array}$ & $\begin{array}{c}\mathrm{N}=14 \text { control } \mathrm{N}=43 \\
\text { training people who use } \\
\text { drugs }\end{array}$ & $\begin{array}{l}\text { Control group received } \\
\text { information about where to } \\
\text { obtain naloxone overdose kits } \\
\text { Training session was a } 30-45 \\
\text { min lecture, detailing signs of } \\
\text { overdose and naloxone use }\end{array}$ & $\begin{array}{l}\mathrm{T}_{0}: 4 / 57 \text { possessed naloxone in the past } \\
\mathrm{T}_{\mathrm{a}}: \text { Improvement in knowledge from } \\
32.6 \text { to } 39.1(p<0.0001) \text { after training } \\
\mathrm{T}_{3 \mathrm{~m}}: \text { Knowledge maintained in trained } \\
\text { group } 38.4(p<0.0001) \\
\text { - Control group also showed } \\
\text { improvement }(32.7 \text { to } 36.3(p<0.005)\end{array}$ & Linden Oaks \\
\hline
\end{tabular}


Table A2. Cont.

\begin{tabular}{|c|c|c|c|c|c|c|}
\hline \multicolumn{7}{|c|}{ Patients' and Bystanders' Perspectives } \\
\hline Author, Title and Journal & Country & Data Collection Method & Participants & Interventions/Study Details & Outcomes & $\begin{array}{c}\text { Funding/ } \\
\text { Sponsorship }\end{array}$ \\
\hline $\begin{array}{l}\text { Madah-Amiri et al., } \\
\qquad 2017 \\
\text { Rapid widespread distribution } \\
\text { of intranasal naloxone for } \\
\text { overdose prevention. Drug } \\
\text { and Alcohol Dependence [40] }\end{array}$ & Norway & $\begin{array}{c}\text { Pre-post questionnaire } \\
\text { (optional): } \\
\mathrm{T}_{0}: \text { Baseline before training } \\
\mathrm{T}_{\mathrm{R}}: \text { Receiving naloxone } \\
\text { refill }\end{array}$ & $\begin{array}{l}\mathrm{N}=1322 \text { nasal naloxone } \\
\text { sprays distributed to } \\
\text { people who use drugs }\end{array}$ & $\begin{array}{l}\text { Training was brief and flexible } \\
\text { individually or in small groups } \\
\text { Training looked at naloxone } \\
\text { administration, assembling the } \\
\text { device, titrating the dose and } \\
\text { administration, rescue } \\
\text { breathing and CPR, aftercare, } \\
\text { side effects and withdrawal } \\
\text { symptoms }\end{array}$ & $\begin{array}{l}\mathrm{T}_{0}: 366 / 433 \text { demonstrated risks factors } \\
\text { for overdose } \\
-361 / 394 \text { ( } 92 \% \text { ) who witnessed an } \\
\text { overdose called EMS } \\
\mathrm{T}_{\mathrm{R}}: 743 / 1322 \text { returned for refill } \\
\text { - 401/743 completed questionnaire } \\
\text { - 277/401 (70\%) used supply on an } \\
\text { overdose, } 265 / 277(96 \%) \text { the victim } \\
\text { survived } \\
-260 / 277(94 \%) \text { used actions taught } \\
-183 / 277(66 \%) \text { called EMS } \\
- \text { “No adverse effects” was the most } \\
\text { common answer for side effects } 76 / 277 \\
(27 \%)\end{array}$ & $\begin{array}{l}\text { Norwegian } \\
\text { Directorate of } \\
\text { Health }\end{array}$ \\
\hline $\begin{array}{c}\text { Maxwell et al., } \\
2006 \\
\text { Prescribing naloxone to } \\
\text { actively injecting heroin users: } \\
\text { a program to reduce heroin } \\
\text { overdose deaths. Journal of } \\
\text { Addictive Diseases [41] }\end{array}$ & US & $\begin{array}{l}\text { Cross-sectional description } \\
\text { of service data }\end{array}$ & $\begin{array}{c}\mathrm{N}=\text { More than } 3500 \\
\text { multi-dose vials of } \\
\text { naloxone were distributed }\end{array}$ & $\begin{array}{c}\text { Education on basic opioid } \\
\text { actions and overdose, risk } \\
\text { factors and prevention } \\
\text { techniques } \\
\text { Physician supplies naloxone }\end{array}$ & $\begin{array}{l}\text { - To date } 319 \text { peer overdose reversals } \\
\text { - One unsuccessful revival has been } \\
\text { reported; however, it was thought to be } \\
\text { attributed to other factors } \\
\text { - In } 5 \text { instances, victim did not respond } \\
\text { until a second injection of naloxone was } \\
\text { given }\end{array}$ & None stated \\
\hline $\begin{array}{c}\text { McAuley et al., } \\
2016 \\
\text { Engagement in a national } \\
\text { naloxone programme among } \\
\text { people who inject drugs. Drug } \\
\text { Alcohol Dependence [61] }\end{array}$ & UK & $\begin{array}{l}\text { Cross-sectional survey pre- } \\
\text { and post-implementation } \\
\text { of a national programme }\end{array}$ & $\begin{array}{c}\text { Approximately } \mathrm{N}= \\
\text { 2000-3000 drug users each } \\
\text { year }\end{array}$ & $\begin{array}{l}\text { Naloxone specific questions } \\
\text { from } 2 \text { Needle Exchange } \\
\text { Surveillance Initiative (NESI) } \\
\text { surveys were analysed }\end{array}$ & $\begin{array}{l}\text { - Participant prescribed naloxone } \\
\text { increased from } 175 / 2146(8 \%) \text { in } \\
2011-2012 \text { to } 745 / 2331(32 \%) \text { in } \\
2013-2014 \\
\text { - Participants who carried naloxone on } \\
\text { them every day decreased from } 27 / 169 \\
(16 \%) \text { in } 2011-2012 \text { to } 39 / 471(5 \%) \text { in } \\
2013-2014\end{array}$ & $\begin{array}{c}\text { Scottish } \\
\text { Government }\end{array}$ \\
\hline $\begin{array}{c}\text { Mueller et al., } \\
2017 \\
\text { Attitudes toward naloxone } \\
\text { prescribing in clinical settings: } \\
\text { a qualitative study of patients } \\
\text { prescribed high dose opioids } \\
\text { for chronic non-cancer pain. } \\
\text { Journal of General Internal } \\
\text { Medicine [52] }\end{array}$ & US & Interviews & $\begin{array}{c}\mathrm{N}=24 \text { chronic opioid users } \\
\text { completed interviews } \\
\text { Mean age } 53.9,66.6 \% \\
\text { females, average length of } \\
\text { opioid prescription } 11.2 \\
\text { years }\end{array}$ & $\begin{array}{l}\text { Patients who received three or } \\
\text { more opioid prescriptions } \\
\text { within 90-day period were } \\
\text { recruited } \\
\text { A priori template of codes was } \\
\text { used as a guide to code the } \\
\text { interviews }\end{array}$ & $\begin{array}{l}\text { - Barriers to naloxone: limited prior } \\
\text { education and knowledge, low } \\
\text { perception of overdose risk, fear of } \\
\text { exacerbating providers' concerns about } \\
\text { opioid misuse and of consequences and } \\
\text { loss of pain treatment if naloxone is used } \\
\text { - Facilitators to naloxone: recognition of } \\
\text { the utility to prevent overdose death, } \\
\text { providers who engaged in empowering } \\
\text { and non-judgmental communication } \\
\text { practices, naloxone training and } \\
\text { education }\end{array}$ & $\begin{array}{l}\text { National } \\
\text { Institute on } \\
\text { Drug Abuse of } \\
\text { the National } \\
\text { Institutes of } \\
\text { Health }\end{array}$ \\
\hline
\end{tabular}


Table A2. Cont.

\begin{tabular}{|c|c|c|c|c|c|c|}
\hline \multicolumn{7}{|c|}{ Patients' and Bystanders' Perspectives } \\
\hline Author, Title and Journal & Country & Data Collection Method & Participants & Interventions/Study Details & Outcomes & $\begin{array}{l}\text { Funding/ } \\
\text { Sponsorship }\end{array}$ \\
\hline $\begin{array}{c}\text { Oliva et al., } \\
2016 \\
\text { Patient perspectives on an } \\
\text { opioid overdose education and } \\
\text { naloxone distribution program } \\
\text { in the U.S. Department of } \\
\text { Veterans Affairs. Substance } \\
\text { Abuse [53] }\end{array}$ & US & Focus groups & $\begin{array}{l}\mathrm{N}=21 \text { substance use } \\
\text { disorder participants }\end{array}$ & $\begin{array}{l}\text { Training was } 1 \mathrm{~h} \text { with } \\
\text { slideshow, videos and } \\
\text { handouts, addressing } \\
\text { epidemiology of overdose } \\
\text { deaths, how to prevent, } \\
\text { recognize and respond to an } \\
\text { overdose including rescue } \\
\text { breathing, naloxone } \\
\text { administration and calling EMS }\end{array}$ & $\begin{array}{l}\text { - Benefits-OEND training is interesting } \\
\text { and novel, the potential to save lives and } \\
\text { empower people } \\
\text { - Concerns-challenges using syringes } \\
\text { (IM kit) } \\
\text { - Differing opinions-naloxone kits may } \\
\text { contribute to relapse, legal and liability } \\
\text { issues, challenges in involving a family } \\
\text { in OEND training, uncertainty about } \\
\text { uses/effects of naloxone }\end{array}$ & $\begin{array}{c}\text { VA Health } \\
\text { Services and } \\
\text { Development's } \\
\text { Quality } \\
\text { Enhancement } \\
\text { Research } \\
\text { Initiative }\end{array}$ \\
\hline $\begin{array}{c}\text { Piper et al., } \\
2008 \\
\text { Evaluation of a naloxone } \\
\text { distribution and administration } \\
\text { program in New York City. } \\
\text { Substance Use and Misuse [42] }\end{array}$ & US & $\begin{array}{c}\text { Post questionnaire: } \\
\mathrm{T}_{\mathrm{R}}: \text { Returning for a refill }\end{array}$ & $\begin{array}{l}\mathrm{N}=122 \text { people who use } \\
\text { opioids }\end{array}$ & $\begin{array}{l}\text { Training program-Skills and } \\
\text { Knowledge on Overdose } \\
\text { Prevention (SKOOP), focused } \\
\text { on overdose prevention } \\
\text { education, naloxone } \\
\text { administration and the } \\
\text { importance of educating family } \\
\text { Physicians provided a } \\
\text { naloxone kit }\end{array}$ & $\begin{array}{l}\mathrm{T}_{\mathrm{R}}: 71 / 122(58.2 \%) \text { witnessed an overdose } \\
\text { since training, and } 50 / 71(70.4 \%) \text { used } \\
\text { naloxone } \\
\text { - In total naloxone was administered } 82 \\
\text { times, } 68 \text { ( } 83 \%) \text { known to live and } 14 \\
(17.1 \%) \text { unknown outcomes } \\
-97 / 122(82.2 \%) \text { felt comfortable using } \\
\text { naloxone }\end{array}$ & $\begin{array}{l}\text { New York City } \\
\text { Council }\end{array}$ \\
\hline $\begin{array}{c}\text { Rowe et al., } \\
2015 \\
\text { Predictors of participant } \\
\text { engagement and naloxone } \\
\text { utilization in a } \\
\text { community-based naloxone } \\
\text { distribution program. } \\
\text { Addiction [43] }\end{array}$ & US & $\begin{array}{c}\text { Pre-post questionnaire } \\
\text { (optional): } \\
\mathrm{T}_{0}: \text { Baseline before training } \\
\mathrm{T}_{\mathrm{R}}: \text { Receiving naloxone } \\
\text { refill }\end{array}$ & $\begin{array}{c}\mathrm{N}=2500 \text { participants who } \\
\text { might witness or } \\
\text { experience an overdose }\end{array}$ & $\begin{array}{l}\text { Analysis of data from DOPE } \\
\text { (Drug Overdose Prevention } \\
\text { Education Project) which } \\
\text { included 5-10 min training and } \\
\text { naloxone kit distribution }\end{array}$ & $\begin{array}{l}\mathrm{T}_{\mathrm{R}}: 702 \text { naloxone administrations } \\
\text { post-training } \\
-192 / 702(27.4 \%) \text { also contacted EMS } \\
\text { - 673/702 }(95.7 \%) \text { known to survive after } \\
\text { naloxone reversal }\end{array}$ & $\begin{array}{l}\text { National } \\
\text { Institutes of } \\
\text { Health }\end{array}$ \\
\hline $\begin{array}{c}\text { Rowe et al., } \\
2016 \\
\text { Neighborhood-level and } \\
\text { spatial characteristics } \\
\text { associated with lay naloxone } \\
\text { reversal events and opioid } \\
\text { overdose deaths. Journal of } \\
\text { Urban Health [62] }\end{array}$ & US & Cross-sectional & $N=316$ overdose reversals & $\begin{array}{c}\text { Locations of all opioid } \\
\text { overdose deaths in San } \\
\text { Francisco from } 2010 \text { to } 2012 \\
\text { were extracted } \\
\text { Naloxone administration } \\
\text { events from } 2010 \text { to } 2012 \\
\text { extracted from the DOPE } \\
\text { project data } \\
\text { Census tract Socioeconomic } \\
\text { status was measured by tract } \\
\text { median income and economic } \\
\text { inequality }\end{array}$ & $\begin{array}{l}\text { - 44/195 (23\%) areas analysed had DOPE } \\
\text { project distribution site } \\
\text { - Distance to nearest DOPE site was not } \\
\text { statistically significantly associated with } \\
\text { number of overdose deaths ( } p=0.093) \\
\text { - Distance to the nearest DOPE site (up } \\
\text { to } 4000 \mathrm{~m} \text { ) was independently } \\
\text { associated with a } 49 \% \text { lower count of } \\
\text { naloxone reversals } p=0.001) \\
\text { - The mean number of reversal events } \\
\text { declined across increasing quartiles of } \\
\text { distance to nearest DOPE site and } \\
\text { median income increased across } \\
\text { quartiles of number of drug arrests, } \\
\text { percentage black or African American } \\
\text { residents, and population density }\end{array}$ & $\begin{array}{l}\text { National } \\
\text { Institutes of } \\
\text { Health }\end{array}$ \\
\hline
\end{tabular}


Table A2. Cont.

\begin{tabular}{|c|c|c|c|c|c|c|}
\hline \multicolumn{7}{|c|}{ Patients' and Bystanders' Perspectives } \\
\hline Author, Title and Journal & Country & Data Collection Method & Participants & Interventions/Study Details & Outcomes & $\begin{array}{l}\text { Funding/ } \\
\text { Sponsorship }\end{array}$ \\
\hline $\begin{array}{c}\text { Seal et al., } \\
2003 \\
\text { Attitudes about prescribing } \\
\text { take-home naloxone to } \\
\text { injection drug users for the } \\
\text { management of heroin } \\
\text { overdose: a survey of } \\
\text { street-recruited injectors in the } \\
\text { San Francisco Bay Area. } \\
\text { Journal of Urban Health: } \\
\text { Bulletin of the New York } \\
\text { Academy of Medicine [63] }\end{array}$ & US & $\begin{array}{l}\text { Qualitative interviews } \\
\text { (themes analysed } \\
\text { quantitatively) }\end{array}$ & $\begin{array}{c}\mathrm{N}=82 \text { people who inject } \\
\text { drugs }\end{array}$ & $\begin{array}{l}\text { Participants were invited from } \\
\text { the Urban Health Study (a } \\
\text { semi-annual study to survey } \\
\text { HIV and hepatitis) based on } \\
\text { their report of heroin overdose }\end{array}$ & $\begin{array}{l}\text { - } 87 \% \text { would participate in a THN } \\
\text { training program } \\
-35 \% \text { believed that having naloxone } \\
\text { would make them feel more comfortable } \\
\text { to use more heroin, the majority did not } \\
\text { feel this way } \\
-62 \% \text { responded they might be less likely } \\
\text { to call EMS if they administer naloxone }\end{array}$ & $\begin{array}{l}\text { Open Society } \\
\text { Institute }\end{array}$ \\
\hline $\begin{array}{c}\text { Seal et al., } \\
2005 \\
\text { Naloxone distribution and } \\
\text { cardiopulmonary resuscitation } \\
\text { training for injection drug } \\
\text { users to prevent heroin } \\
\text { overdose death: a pilot } \\
\text { intervention study. Journal of } \\
\text { Urban Health [44] }\end{array}$ & US & $\begin{array}{c}\text { Post interview: } \\
\mathrm{T}_{1-6 \mathrm{~m}} \text { : Monthly for } 6 \\
\text { months post-training }\end{array}$ & $\begin{array}{c}\mathrm{N}=24 \text { people who inject } \\
\text { drugs }\end{array}$ & $\begin{array}{c}\text { Training involved four, } \\
\text { two-hour interactive sessions. } \\
\text { 1: recognize overdose and } \\
\text { overdose prevention 2: CPR } \\
\text { practice 3: accessing medical } \\
\text { services 4: naloxone } \\
\text { administration Naloxone kit } \\
\text { was dispensed after } \\
\text { completion of training }\end{array}$ & $\begin{array}{l}\mathrm{T}_{1-6 \mathrm{~m}}: 20 \text { overdoses were witnessed, } \\
\text { naloxone was used in } 15 \text { cases and there } \\
\text { was some form of other intervention in } \\
\text { all } 20 \text { cases } \\
\text { - EMS was contacted in } 6 / 20 \text { cases } \\
\text { - Reasons for not contacting was fear of } \\
\text { police in } 10 \text { cases, no phone in } 5 \text { cases } \\
\text { and no perceived need in } 5 \text { cases } \\
\mathrm{T}_{6 \mathrm{~m}}: \text { Knowledge of overdose had } \\
\text { increased } \\
\text {-Frequency of injecting heroin decreased } \\
\text { with people injecting } 90+\text { times in a } \\
\text { month from } 11 / 24 \text { to } 0 / 24(p=0.003)\end{array}$ & None stated \\
\hline $\begin{array}{c}\text { Sherman et al., } \\
2008 \\
\text { A qualitative study of overdose } \\
\text { responses among Chicago } \\
\text { IDUs. Harm Reduction Journal } \\
\text { [45] } \\
\end{array}$ & US & Qualitative interviews & $\begin{array}{l}\mathrm{N}=31 \text { injecting drug users } \\
81 \% \text { male, median age } 38 \\
81 \% \text { injected opioids daily }\end{array}$ & $\begin{array}{l}\text { Semi-structured qualitative } \\
\text { interviews with participants } \\
\text { recommended by needle } \\
\text { exchange staff }\end{array}$ & $\begin{array}{l}\text { - All cases of naloxone use were } \\
\text { successful } \\
\text { - Participants were comfortable and } \\
\text { confident } \\
\text { - Unanimous fear of contacting } 911 \text { due } \\
\text { to fear of police involvement }\end{array}$ & $\begin{array}{c}\text { Tides } \\
\text { Foundation }\end{array}$ \\
\hline $\begin{array}{c}\text { Strang et al., } \\
2008 \\
\text { Overdose training and } \\
\text { take-home naloxone for opiate } \\
\text { users: prospective cohort study } \\
\text { of impact on knowledge and } \\
\text { attitudes and subsequent } \\
\text { management of overdoses. } \\
\text { Addiction [46] }\end{array}$ & UK & $\begin{array}{c}\text { Pre-post questionnaire: } \\
\mathrm{T}_{0} \text { : Baseline before training } \\
\mathrm{T}_{\mathrm{a}} \text { : Immediately after } \\
\text { training } \\
\text { Interview } \\
\mathrm{T}_{2 \mathrm{~m}}: 2 \text { months }\end{array}$ & $\mathrm{N}=239$ opiate users & $\begin{array}{l}\text { Training focused on risk } \\
\text { factors, recognition and actions } \\
\text { to be taken in an overdose, } \\
\text { including naloxone } \\
\text { administration Participants } \\
\text { were provided with naloxone }\end{array}$ & $\begin{array}{l}\mathrm{T}_{0} \text { : Overall knowledge of overdose } \\
\text { mean score was } 16.7 / 26 \text { and at } \mathrm{T}_{\mathrm{a}}: 21.4 / 26 \\
(p<0.001) \\
-151 / 196(77 \%) \text { willing to administer } \\
\text { naloxone and at } \mathrm{T}_{\mathrm{a}} \text { : increase to } 192 / 194 \\
(99 \%) \\
\mathrm{T}_{2 \mathrm{~m}}: 169 / 173(98 \%) \text { reported confidence } \\
\text { in ability to recognize opiate overdose } \\
\text { and } 168 / 172(97 \%) \text { confident in their } \\
\text { ability to manage it } \\
-11 \text { naloxone administrations } \\
\text { - No formal adverse events reported }\end{array}$ & $\begin{array}{l}\text { National } \\
\text { Treatment } \\
\text { Agency for } \\
\text { Substance } \\
\text { Misuse }\end{array}$ \\
\hline
\end{tabular}


Table A2. Cont.

\begin{tabular}{|c|c|c|c|c|c|c|}
\hline \multicolumn{7}{|c|}{ Patients' and Bystanders' Perspectives } \\
\hline Author, Title and Journal & Country & Data Collection Method & Participants & Interventions/Study Details & Outcomes & $\begin{array}{l}\text { Funding/ } \\
\text { Sponsorship }\end{array}$ \\
\hline $\begin{array}{c}\text { Strang et al., } \\
1999 \\
\text { Preventing opiate overdose } \\
\text { fatalities with take-home } \\
\text { naloxone: Pre-launch study of } \\
\text { possible impact and } \\
\text { acceptability. Addiction [57] }\end{array}$ & UK & $\begin{array}{l}\text { Interviews analysed } \\
\text { quantitatively }\end{array}$ & $\begin{array}{c}\mathrm{N}=312 \text { community sample } \\
\text { (people who inject drugs) } \\
\text { Mean age } 30.6, \text { age first } \\
\text { injected } 20.1,37.2 \% \text { women } \\
\mathrm{N}=142 \text { treatment sample } \\
\text { (people who inject drugs) } \\
\text { Mean age } 35.8, \text { age first } \\
\text { injected } 19.5,26.8 \% \text { women }\end{array}$ & $\begin{array}{l}\text { Community sample (control) } \\
\text { and treatment sample } \\
\text { (Methadone participants) } \\
\text { Treatment sample interviews } \\
\text { were conducted after } \\
\text { community sample analysis in } \\
\text { order to address any topics } \\
\text { brought up by the community } \\
\text { sample }\end{array}$ & $\begin{array}{l}\text { - } 49 / 142(35 \%) \text { had heard of naloxone } \\
\text { - } 90 / 142(70 \%) \text { of treatment group } \\
\text { considered naloxone distribution a good } \\
\text { idea } \\
-9 / 142(6 \%) \text { reported it may increase } \\
\text { their heroin dose } \\
\text { - Estimated that } 2 / 3 \text { of the } 69 \text { overdose } \\
\text { fatalities reported by participants could } \\
\text { have bene prevented }\end{array}$ & None stated \\
\hline $\begin{array}{l}\text { Tobin et al., } \\
2009 \\
\text { Evaluation of the staying alive } \\
\text { programme: training injection } \\
\text { drug users to properly } \\
\text { administer naloxone and save } \\
\text { lives. International Journal of } \\
\text { Drug Policy [72] }\end{array}$ & US & $\begin{array}{l}\text { Pre-post questionnaire: } \\
\mathrm{T}_{0} \text { : Baseline before training } \\
\mathrm{T}_{6 \mathrm{~m}}: \text { 6-month follow up }\end{array}$ & $\begin{array}{c}\mathrm{N}=250 \text { people with opioid } \\
\text { addiction }\end{array}$ & $\begin{array}{l}\text { Training details were not } \\
\text { discussed }\end{array}$ & $\begin{array}{l}\mathrm{T}_{0} \text { : No one had used naloxone } \\
\mathrm{T}_{6 \mathrm{~m}}: 19 \text { reported using naloxone } \\
\text { - Knowledge improved for } 46 \% \text { of the } \\
\text { sample about risk of relapse after } \\
\text { naloxone, } 35 \% \text { did not know at either } \\
\text { time point or knowledge decreased } \\
-25 \% \text { reported increase comfort in } \\
\text { managing overdose post-training and } \\
32 \% \text { were negative or unsure at both } \\
\text { time points }\end{array}$ & $\begin{array}{l}\text { National } \\
\text { Institute on } \\
\text { Drug Use }\end{array}$ \\
\hline $\begin{array}{c}\text { Wagner et al., } \\
2010 \\
\text { Evaluation of an overdose } \\
\text { prevention and response } \\
\text { training programme for } \\
\text { injection drug users in the skid } \\
\text { row area of Los Angeles, CA. } \\
\text { International Journal of Drug } \\
\text { Policy [48] }\end{array}$ & US & $\begin{array}{l}\text { Pre-post questionnaire: } \\
\mathrm{T}_{0} \text { : Baseline before training } \\
\mathrm{T}_{3 \mathrm{~m}}: \text { 3-month follow up }\end{array}$ & $\begin{array}{c}\mathrm{N}=66 \text { people who inject } \\
\text { drugs }\end{array}$ & $\begin{array}{l}\text { Training was individual or in } \\
\text { small groups for } 1 \mathrm{~h} \text { addressing } \\
\text { opioid overdose mechanism, } \\
\text { prevention, recognition and } \\
\text { response } \\
\text { Physician dispensed naloxone } \\
\text { after training completion }\end{array}$ & $\begin{array}{l}\mathrm{T}_{3 \mathrm{~m}} \text { : Overall knowledge increased from } \\
77 \%-92 \%(p<0.0001) \\
-53 \% \text { reported decrease in drug use } \\
\text { - No significant change in attitudes about } \\
\text { overdose response } \\
\text { - Number of recommended response } \\
\text { techniques increased from } 2 \text { to } 3.3(p= \\
0.01)\end{array}$ & $\begin{array}{l}\text { "foundation } \\
\text { support and } \\
\text { private } \\
\text { fundraising" }\end{array}$ \\
\hline $\begin{array}{c}\text { Walley et al., } \\
2013 \\
\text { Opioid overdose rates and } \\
\text { implementation of overdose } \\
\text { education and nasal naloxone } \\
\text { distribution in Massachusetts: } \\
\text { interrupted time series analysis. } \\
\text { BMJ [49] }\end{array}$ & US & Cross-sectional survey & $\begin{array}{c}\mathrm{N}=4857 \text { people who inject } \\
\text { drugs or bystanders } \\
\text { enrolled in naloxone } \\
\text { training }\end{array}$ & $\begin{array}{l}\text { Trainings from } 10-60 \mathrm{~min} \\
\text { about risk factors of overdose } \\
\text { and naloxone administration } \\
\text { Baseline and refill } \\
\text { questionnaires were obtained } \\
\text { from the Massachusetts } \\
\text { Department of Public Health } \\
\text { OEND program (2006-2009) }\end{array}$ & $\begin{array}{l}\text { - } 327 \text { rescue attempts 150/153 (98\%) used } \\
\text { naloxone successfully } \\
\text { - Decrease in opioid-related deaths in } \\
\text { communities with OEND }\end{array}$ & $\begin{array}{c}\text { Center for } \\
\text { Disease Control } \\
\text { and Prevention }\end{array}$ \\
\hline
\end{tabular}


Table A2. Cont.

\begin{tabular}{|c|c|c|c|c|c|c|}
\hline \multicolumn{7}{|c|}{ Patients' and Bystanders' Perspectives } \\
\hline Author, Title and Journal & Country & Data Collection Method & Participants & Interventions/Study Details & Outcomes & $\begin{array}{l}\text { Funding/ } \\
\text { Sponsorship }\end{array}$ \\
\hline $\begin{array}{l}\text { Williams et al., } \\
2014 \\
\text { Training family members to } \\
\text { manage heroin overdose and } \\
\text { administer naloxone: } \\
\text { randomized trial of effects on } \\
\text { knowledge and attitudes. } \\
\text { Addiction [50] }\end{array}$ & UK & $\begin{array}{c}\mathrm{RCT} \\
\text { Assessment: } \\
\mathrm{T}_{\mathrm{a}}: \text { Immediately after } \\
\text { training } \\
\mathrm{T}_{3 \mathrm{~m}}: 3 \text { months } \\
\text { post-training }\end{array}$ & $\begin{array}{l}\mathrm{N}=87 \text { experimental group } \\
\mathrm{N}=75 \text { control family } \\
\text { members and carers }\end{array}$ & $\begin{array}{c}\text { Opioid overdose } \\
\text { knowledge/attitude scale } \\
\text { (OOKS and OOAS) were } \\
\text { developed } \\
\text { Training (60 minute) on } \\
\text { overdose risk factors, } \\
\text { recognition, actions to take, } \\
\text { what naloxone is and how to } \\
\text { use it } \\
\text { Control group did not receive } \\
\text { training }\end{array}$ & $\begin{array}{l}-\mathrm{T}_{3 \mathrm{~m}} \text { : Control group had an } 11 \% \\
\text { increase in knowledge and } 35 \% \text { increase } \\
\text { for training } \\
\text { - Control group had a } 20 \% \text { increase in } \\
\text { positive attitude scored and } 54 \% \text { for } \\
\text { training group. }\end{array}$ & $\begin{array}{l}\text { Alban } \\
\text { Programme and } \\
\text { Institute of } \\
\text { Social } \\
\text { Psychiatry and } \\
\text { University of } \\
\text { London }\end{array}$ \\
\hline $\begin{array}{l}\text { Worthington et al., } \\
2006 \\
\text { Opiate users' knowledge about } \\
\text { overdose prevention and } \\
\text { naloxone in New York City: a } \\
\text { focus group study. Harm } \\
\text { Reduction Journal [54] }\end{array}$ & US & Focus groups & $\begin{array}{c}\mathrm{N}=8 \text { people who use } \\
\text { opioids } \\
\text { 1st focus group } \\
\text { 37\% African American } \\
\text { females, } 37 \% \text { white males } \\
\mathrm{N}=5 \text { people who use } \\
\text { opioids in the 2nd focus } \\
\text { group } 80 \% \text { white males }\end{array}$ & $\begin{array}{l}\text { Recruited by flyers, word of } \\
\text { mouth etc, from Lower East } \\
\text { Side Harm Reduction Center } \\
\text { (LESHR) discussing overdose } \\
\text { experience especially with } \\
\text { naloxone and comfort with } \\
\text { naloxone }\end{array}$ & $\begin{array}{l}\text { - Both groups expressed some hesitation } \\
\text { about take-home naloxone } \\
-4 \text { major themes addressed: support for } \\
\text { naloxone as a lifesaving measure, } \\
\text { challenges of administering naloxone } \\
\text { during an overdose, fear of } \\
\text { dopesickness and police arrest }\end{array}$ & $\begin{array}{c}\text { Tides } \\
\text { Foundation }\end{array}$ \\
\hline $\begin{array}{l}\text { Wright et al., } \\
2006 \\
\text { Homeless drug users' } \\
\text { awareness and risk perception } \\
\text { of peer "Take Home Naloxone" } \\
\text { use-a qualitative study. } \\
\text { Substance Abuse Treatment, } \\
\text { Prevention, and Policy [55] }\end{array}$ & UK & Qualitative interviews & $\begin{array}{l}\mathrm{N}=27 \text { people who use } \\
\text { heroin } \\
19 \text { men and } 8 \text { women }\end{array}$ & $\begin{array}{l}\text { Participants recruited from } 3 \\
\text { sites who deliver services to } \\
\text { homeless people who use } \\
\text { drugs }\end{array}$ & $\begin{array}{l}\text { - Participants had good prior knowledge } \\
\text { about naloxone; however, many mistook } \\
\text { it for adrenaline } \\
\text { - Clear willingness to administer THN if } \\
\text { required } \\
\text { - Concerns about misuse and malicious } \\
\text { use } \\
\text { - Participants often saw it as obviating } \\
\text { the need to call EMS } \\
\text { - Some participants revealed that current } \\
\text { information from professionals is often } \\
\text { not appealing or likely to increase their } \\
\text { knowledge, other saw a role for } \\
\text { professionals in education }\end{array}$ & None stated \\
\hline $\begin{array}{c}\text { Yorkell et al., } \\
2011 \\
\text { Opioid overdose prevention } \\
\text { and naloxone distribution in } \\
\text { Rhode Island. Medicine and } \\
\text { Health, Rhode Island [51] }\end{array}$ & US & $\begin{array}{l}\text { Pre-post report about use: } \\
\mathrm{T}_{3 \mathrm{~m}} \text { : } 3 \text {-month follow up } \\
\text { OR after } 1^{\text {st }} \text { naloxone use } \\
\text { (whichever comes first) }\end{array}$ & $\begin{array}{c}\mathrm{N}=120 \text { people who use } \\
\text { opioids trained in naloxone } \\
\text { use }\end{array}$ & $\begin{array}{l}\text { Interactive training process } \\
\text { about overdose causes, } \\
\text { prevention and IM naloxone } \\
\text { administration } \\
\text { Physician distributed naloxone }\end{array}$ & $\begin{array}{l}T_{3 m}: 10 \text { individuals at follow up } \\
-5 / 10 \text { used naloxone successfully } \\
-5 / 10 \text { used other techniques successfully }\end{array}$ & $\begin{array}{l}\text { CDC/NCIPC, } \\
\text { NIH/CFAR, } \\
\text { CDARR }\end{array}$ \\
\hline
\end{tabular}

US = United States, UK = United Kingdom, EMS = Emergency Medical Services, THN = Take-Home Naloxone. 
Table A3. Summary of Included Studies from Professionals' Perspectives.

\begin{tabular}{|c|c|c|c|c|c|c|}
\hline \multicolumn{7}{|c|}{ Professionals' Perspective } \\
\hline Author, Title and Journal & Country & Data Collection Method & Participants & Interventions/Study Details & Outcomes & $\begin{array}{l}\text { Funding/ } \\
\text { Sponsorship }\end{array}$ \\
\hline $\begin{array}{c}\text { Ashrafioun et al., } \\
\quad 2016 \\
\text { Evaluation of knowledge and } \\
\text { confidence following opioid } \\
\text { overdose prevention training: } \\
\text { a comparison of types of } \\
\text { training participants and } \\
\text { naloxone administration } \\
\text { method. Substance Abuse [75] }\end{array}$ & US & $\begin{array}{c}\text { Assessment: } \\
\mathrm{T}_{0} \text { : Baseline before training } \\
\mathrm{T}_{\mathrm{a}} \text { : Immediately after } \\
\text { training }\end{array}$ & $\begin{array}{c}\mathrm{N}=93 \text { medical providers } \\
\mathrm{N}=40 \text { family and friends } \\
\mathrm{N}=3 \text { patients } \mathrm{N}=139 \\
\text { other }\end{array}$ & $\begin{array}{l}\text { Training was } 20-45 \text { min on } \\
\text { opioid overdose epidemiology, } \\
\text { physiology and risk factors, } \\
\text { naloxone's role in overdose, its } \\
\text { outcomes and how to use it } \\
\text { both IM and IN }\end{array}$ & $\begin{array}{l}\text { - All groups knowledge } 83 \% \text { increase } \\
\text { and confidence } 85 \% \text { from pre-training } \\
(\mathrm{M}=4.2, \mathrm{SD}=1.1 \text { and } \mathrm{M} 3.7, \mathrm{SD} 1.8) \text { to } \\
\text { post-( } \mathrm{M}=6.2, \mathrm{SD} 1 \text { and } \mathrm{M}=5.9, \mathrm{SD}= \\
1.2) \\
\text { - Confidence and knowledge did not } \\
\text { vary by route of administration } \\
\text { - No difference in pre- or post-knowledge } \\
\text { scores for participant type }\end{array}$ & $\begin{array}{c}\text { Office of } \\
\text { Academic } \\
\text { Affiliations, } \\
\text { Advanced } \\
\text { Fellowship } \\
\text { Program in the } \\
\text { Mental Illness } \\
\text { Research and } \\
\text { Treatment, } \\
\text { Department of } \\
\text { Veterans Affairs }\end{array}$ \\
\hline $\begin{array}{l}\text { Bailey and Wermeling } \\
2014 \\
\text { Naloxone for opioid overdose } \\
\text { prevention: pharmacists' role } \\
\text { in community-based practice } \\
\text { settings. Annals of } \\
\text { Pharmacotherapy [64] }\end{array}$ & US & Interviews & $\mathrm{N}=6$ pharmacists & $\begin{array}{l}\text { Participants from community } \\
\text { and clinic-based settings were } \\
\text { identified through their } \\
\text { affiliations with collaborating } \\
\text { providers }\end{array}$ & $\begin{array}{l}\text { - Providers found a need for a } \\
\text { prevention strategy for patients that they } \\
\text { see on a day-to-day basis } \\
\text { - Many pharmacies have developed their } \\
\text { own educational protocols for patients } \\
\text { - Barriers: financial and reimbursement, } \\
\text { state regulations }\end{array}$ & $\begin{array}{l}\text { National Center } \\
\text { for Research } \\
\text { Resources and } \\
\text { the National } \\
\text { Center for } \\
\text { Advancing } \\
\text { Translational } \\
\text { Sciences }\end{array}$ \\
\hline $\begin{array}{c}\text { Behar et al., } \\
2017 \\
\text { Acceptability of naloxone } \\
\text { co-prescription among primary } \\
\text { care providers treating patients } \\
\text { on long-term opioid therapy } \\
\text { for pain. Journal of General } \\
\text { Internal Medicine [65] }\end{array}$ & US & Cross-sectional survey & $\begin{array}{l}\mathrm{N}=111 \text { safety-net primary } \\
\text { care prescribers who } \\
\text { initiated naloxone } \\
\text { co-prescribing } \\
50 \% \text { had been practicing for } \\
\text { less than } 5 \text { years, most say } \\
10 \text { or less patients on } \\
\text { opioids per month }\end{array}$ & $\begin{array}{l}\text { Anonymous survey took five } \\
\text { minutes to complete } \\
\text { Physicians recruited via email }\end{array}$ & $\begin{array}{l}-79.3 \% \text { had co-prescribed naloxone } \\
\text { since program initiation } \\
-99.1 \% \text { reported they were likely to } \\
\text { co-prescribe naloxone } \\
-3.6 \% \text { suggested naloxone prescribing } \\
\text { may lead to increase in opioid } \\
\text { prescribing } \\
\text { - Most frequent concerns around patient } \\
\text { education }\end{array}$ & $\begin{array}{l}\text { National } \\
\text { Institutes of } \\
\text { Health }\end{array}$ \\
\hline $\begin{array}{c}\text { Beletsky et al., } \\
2007 \\
\text { Physicians' knowledge of and } \\
\text { willingness to prescribe } \\
\text { naloxone to reverse accidental } \\
\text { opiate overdose: challenges } \\
\text { and opportunities. Journal of } \\
\text { Urban Health [92] }\end{array}$ & US & Cross-sectional survey & $\begin{array}{c}\mathrm{N}=563 \text { physicians } \\
70 \% \text { males, } 83 \% \text { white }\end{array}$ & $\begin{array}{c}\text { Recruited from American } \\
\text { Medical Association } \\
\text { Physicians were mailed the } \\
\text { survey and followed up with a } \\
\text { phone call } \\
\text { The survey assessed attitudes } \\
\text { towards injecting drug users }\end{array}$ & $\begin{array}{l}\text { - 129/563 (23\%) had heard of prescribing } \\
\text { naloxone as a prevention measure } \\
-54 \% \text { indicated that they would never } \\
\text { "consider prescribing naloxone and } \\
\text { explaining its use to an IDU patient" } \\
\text { - Mean age of } 51 \text { rather than } 48 \text { was } \\
\text { associated with having heard of this } \\
\text { intervention }\end{array}$ & $\begin{array}{l}\text { Substance } \\
\text { Abuse Policy } \\
\text { Research } \\
\text { Program of the } \\
\text { Robert Wood } \\
\text { Johnson } \\
\text { Foundation }\end{array}$ \\
\hline
\end{tabular}


Table A3. Cont.

\begin{tabular}{|c|c|c|c|c|c|c|}
\hline \multicolumn{7}{|c|}{ Professionals' Perspective } \\
\hline Author, Title and Journal & Country & Data Collection Method & Participants & Interventions/Study Details & Outcomes & $\begin{array}{l}\text { Funding/ } \\
\text { Sponsorship }\end{array}$ \\
\hline $\begin{array}{c}\text { Coffin et al., } \\
2003 \\
\text { Preliminary evidence of } \\
\text { healthcare provider support for } \\
\text { naloxone prescription as } \\
\text { overdose fatality prevention } \\
\text { strategy in New York City. } \\
\text { Journal of Urban Health [96] }\end{array}$ & US & Cross-sectional survey & $\begin{array}{c}\mathrm{N}=363 \text { physicians } \\
54 \% \text { male, } 68.4 \% \text { white, } \\
68 \% \mathrm{MD} \text { and } 15.6 \% \mathrm{NP}, \\
39.6 \% \text { practice in an office } \\
\text { and } 32.1 \% \text { in a hospital }\end{array}$ & $\begin{array}{c}\text { Participants recruited from a } \\
\text { list of prescribers obtained } \\
\text { from the New York } \\
\text { Department of Education } \\
7-10 \text { min survey measured } \\
\text { attitudes around injecting drug } \\
\text { users }\end{array}$ & $\begin{array}{l}\text { - } 33.4 \% \text { of respondents reported that they } \\
\text { would consider prescribing naloxone to } \\
\text { patients at risk of opiate overdose; } 29.4 \% \\
\text { were unsure, and } 37.1 \% \text { would not }\end{array}$ & None stated \\
\hline $\begin{array}{c}\text { Dahlem et al., } \\
2016 \\
\text { Development and } \\
\text { implementation of intranasal } \\
\text { naloxone opioid overdose } \\
\text { response protocol at a } \\
\text { homeless health clinic. Journal } \\
\text { of the American Association of } \\
\text { Nurse Practitioners [76] }\end{array}$ & US & $\begin{array}{c}\text { Intervention and } \\
\text { post-survey: } \\
\mathrm{T}_{\mathrm{a}}: \text { Immediately after } \\
\text { training }\end{array}$ & $\begin{array}{c}\mathrm{N}=35 \text { homeless shelter } \\
\text { staff }\end{array}$ & $\begin{array}{l}\text { Overdose education and } \\
\text { naloxone distribution flow } \\
\text { chart was modified for } \\
\text { non-healthcare staff and again } \\
\text { for healthcare staff } \\
\text { 1-2 h training in assembly and } \\
\text { administration of IN naloxone, } \\
\text { both didactic and hands on }\end{array}$ & $\begin{array}{l}\mathrm{T}_{\mathrm{a}} \text { : Training was reported average to } \\
\text { excellent (mean score } 4.54 / 5) \\
\text { - All learning outcomes were met } \\
\text { - Participants suggested training be } \\
\text { annually }(19 / 35,54 \%) \text { or biannually } \\
(15 / 35,43 \%)\end{array}$ & None stated \\
\hline $\begin{array}{c}\text { Drainoni et al., } \\
2016 \\
\text { Why is it so hard to implement } \\
\text { change? A qualitative } \\
\text { examination of barriers and } \\
\text { facilitators to distribution of } \\
\text { naloxone for overdose } \\
\text { prevention in a safety net } \\
\text { environment. BMC Research } \\
\text { Notes [66] }\end{array}$ & US & $\begin{array}{l}\text { Interviews and focus } \\
\text { groups }\end{array}$ & $\begin{array}{c}\mathrm{N}=19 \text { physicians } \\
\mathrm{N}=26 \text { registered nurses } \\
\mathrm{N}=3 \text { HPS } \\
\mathrm{N}=2 \text { pharmacists } \\
56 \% \text { female, } 34 \% \text { over } 25 \\
32 \% 2-5 \text { years working in } \\
\text { ED }\end{array}$ & $\begin{array}{l}\text { All participants recruited from } \\
\text { the ED department } \\
\text { Interviews from } 25-36 \mathrm{~min}, \\
\text { about barriers to new policy to } \\
\text { increase naloxone distribution } \\
\text { in emergency department }\end{array}$ & $\begin{array}{l}\text { - Facilitators included: the intervention } \\
\text { being real-world driven with leadership } \\
\text { support, education and training efforts, } \\
\text { availability of resources and ability to } \\
\text { leave the ED with the naloxone kit in } \\
\text { hand } \\
\text { - Barriers: protocols and policy-related } \\
\text { barriers, workflow and logistics, } \\
\text { patient-related (stigma), staff roles and } \\
\text { responsibilities education and training }\end{array}$ & $\begin{array}{c}\text { Established } \\
\text { Investigator } \\
\text { Innovation } \\
\text { Award from the } \\
\text { Boston } \\
\text { University } \\
\text { School of Public } \\
\text { Health }\end{array}$ \\
\hline $\begin{array}{c}\text { Gatewood et al., } \\
2016 \\
\text { Academic physicians' and } \\
\text { medical students' perceived } \\
\text { barriers toward bystander } \\
\text { administered naloxone as an } \\
\text { overdose prevention strategy. } \\
\text { Addictive Behaviors [67] }\end{array}$ & US & $\begin{array}{l}\text { Interviews and discussion } \\
\text { groups }\end{array}$ & $\begin{array}{c}N=5 \text { physicians } \\
N=25 \text { medical students }\end{array}$ & $\begin{array}{l}\text { Participants were recruited due } \\
\text { to their affiliation with } 2 \\
\text { Baltimore medical schools } \\
\text { Interviews and discussion } \\
\text { groups were tailored to } \\
\text { physician or student }\end{array}$ & $\begin{array}{l}\text { - Barriers in prescribing naloxone to } \\
\text { bystanders: duration of action of } \\
\text { naloxone, route of administration, lack } \\
\text { of knowledge about safety outside } \\
\text { hospital setting, little basis for patient } \\
\text { follow up, insult patients whom are not } \\
\text { "addicted" but are at risk, "enabling" } \\
\text { addicts to use more } \\
\text { - Withdrawal symptoms, stigma } \\
\text { associated with carrying naloxone }\end{array}$ & None stated \\
\hline
\end{tabular}


Table A3. Cont.

\begin{tabular}{|c|c|c|c|c|c|c|}
\hline \multicolumn{7}{|c|}{ Professionals' Perspective } \\
\hline Author, Title and Journal & Country & Data Collection Method & Participants & Interventions/Study Details & Outcomes & $\begin{array}{l}\text { Funding/ } \\
\text { Sponsorship }\end{array}$ \\
\hline $\begin{array}{l}\text { Green et al., } \\
\qquad 2013 \\
\text { Barriers to medical provider } \\
\text { support for prescription } \\
\text { naloxone as overdose antidote } \\
\text { for lay responders. Substance } \\
\text { use and misuse [68] }\end{array}$ & US & Qualitative interviews & $\begin{array}{c}\mathrm{N}=4 \text { emergency } \\
\text { department } \\
\text { providers } \\
\mathrm{N}=9 \text { substance use } \\
\text { treatment providers } \\
\mathrm{N}=6 \text { pain specialists } \\
\mathrm{N}=5 \text { family medicine } \\
\text { practitioners including } \\
\text { doctors, } \\
\text { nurses, and a physician } \\
\text { assistant }\end{array}$ & $\begin{array}{l}\text { Interviewees were nominated } \\
\text { by member of the studies } \\
\text { advisory board and then } \\
\text { interviewees could further } \\
\text { recommend participants }\end{array}$ & $\begin{array}{l}\text { - Barriers: "Safety Net" effect allowing } \\
\text { for risker drug use, the need to train the } \\
\text { "Right People" with proper education } \\
\text { and not a stand-alone solution }\end{array}$ & $\begin{array}{c}\text { Centers for } \\
\text { Disease Control } \\
\text { and Prevention }\end{array}$ \\
\hline $\begin{array}{c}\text { Leece et al., } \\
2015 \\
\text { Can naloxone prescription and } \\
\text { overdose training for opioid } \\
\text { users work in family practice? } \\
\text { Perspectives of family } \\
\text { physicians. Canadian Family } \\
\text { Physician [69] } \\
\end{array}$ & Canada & $\begin{array}{l}\text { Focus groups and } \\
\text { workshops }\end{array}$ & $\mathrm{N}=17$ family physicians & $\begin{array}{l}\text { Two hour workshops and focus } \\
\text { groups with physicians } \\
\text { attending College of Family } \\
\text { Physicians of Canada } \\
\text { conference } \\
\text { Focus groups performed a } \\
\text { SWOT analysis }\end{array}$ & $\begin{array}{l}\text { - Facilitators: safety, setting, engagement } \\
\text { and education, logistics, and evidence } \\
\text { - Barriers: guidelines and } \\
\text { implementation, medicolegal } \\
\text { uncertainties, support, equity or stigma, } \\
\text { and evidence }\end{array}$ & None stated \\
\hline $\begin{array}{c}\text { Nielsen et al., } \\
2016 \\
\text { Community pharmacist } \\
\text { knowledge, attitudes and } \\
\text { confidence regarding naloxone } \\
\text { for overdose reversal. } \\
\text { Addiction [70] }\end{array}$ & Australia & Cross-sectional survey & $\begin{array}{c}\mathrm{N}=595 \text { pharmacists } \\
\text { Average time practicing } 6.5 \\
\text { years, } 54.5 \% \text { male, } 51.1 \% \\
\text { aged } 25-34\end{array}$ & $\begin{array}{l}\text { Random sample of pharmacies } \\
\text { generated from an existing list } \\
\text { of Australian community } \\
\text { pharmacies and surveys were } \\
\text { administered online }\end{array}$ & $\begin{array}{l}\text { - Mean attitude score } 6.18 \text {, reflecting } \\
\text { positive attitudes toward } \\
\text { harm-reduction services } \\
-190 / 595(31.9 \%) \text { were confident in } \\
\text { educating about naloxone use } \\
\text { - Lowest levels of comfort reported in } \\
\text { supplying naloxone to people buying } \\
\text { syringes }(68.4 \%) \text { and opioid substitution } \\
\text { therapy }(77.6 \%) \\
\text { - Greater support for overdose was } \\
\text { associated with whether the pharmacy } \\
\text { provided OST ( } p=0.004) \\
-136 / 595(23 \%) \text { pharmacies stored } \\
\text { naloxone } \\
\text { - Mean score in naloxone knowledge test } \\
1.8 / 5 \\
\text { - Barriers included time involved, lack of } \\
\text { training and knowledge of state laws } \\
\text { and lack of reimbursement for patient } \\
\text { education and counselling }\end{array}$ & $\begin{array}{l}\text { Substance } \\
\text { Misuse } \\
\text { Prevention and } \\
\text { Service } \\
\text { Improvements } \\
\text { Grant }\end{array}$ \\
\hline
\end{tabular}


Table A3. Cont.

\begin{tabular}{|c|c|c|c|c|c|c|}
\hline \multicolumn{7}{|c|}{ Professionals' Perspective } \\
\hline Author, Title and Journal & Country & Data Collection Method & Participants & Interventions/Study Details & Outcomes & $\begin{array}{l}\text { Funding/ } \\
\text { Sponsorship }\end{array}$ \\
\hline $\begin{array}{l}\text { Sondhi et al., } \\
2016 \\
\text { Stakeholder perceptions and } \\
\text { operational barriers in the } \\
\text { training and distribution of } \\
\text { take-home naloxone within } \\
\text { prisons in England. Harm } \\
\text { Reduction Journal [71] }\end{array}$ & UK & Qualitative interviews & $\begin{array}{c}\mathrm{N}=17 \text { key stakeholders } \\
\mathrm{N}=26 \text { prisoners }\end{array}$ & $\begin{array}{l}\text { Both formal, structured and } \\
\text { informal discussions with a } \\
\text { range of stakeholders } \\
\text { including prison } \\
\text { representatives from healthcare } \\
\text { and substance misuse services, } \\
\text { strategic leads for NHS } \\
\text { England and representatives } \\
\text { from Martindale Pharma } \\
\text { Four focus groups of male } \\
\text { prisoners about views on } \\
\text { participating in training }\end{array}$ & $\begin{array}{l}\text { - Prisoners were sometimes confused } \\
\text { about the message of THN as most } \\
\text { primary prison goals were to abstain } \\
\text { from opioid use } \\
\text { - Staff found a disconnect between THN } \\
\text { as harm reduction and potential } \\
\text { incentive for using drugs } \\
\text { - Staff had concerns about who and } \\
\text { when to give THN training, the logistical } \\
\text { issues of distributing THN within a } \\
\text { prison setting and the need for a "whole } \\
\text { systems" approach, involving senior } \\
\text { prison staff. }\end{array}$ & $\begin{array}{l}\text { National Health } \\
\text { Service England }\end{array}$ \\
\hline $\begin{array}{l}\text { Tobin et al., } \\
2005 \\
\text { Attitudes of emergency } \\
\text { medical service providers } \\
\text { towards naloxone distribution } \\
\text { programs. Journal of Urban } \\
\text { Health [72] }\end{array}$ & US & Cross-sectional survey & $\begin{array}{c}\mathrm{N}=154 \text { emergency medical } \\
\text { service providers } \\
79 \% \text { male, } 75 \% \text { white, mean } \\
\text { age } 34\end{array}$ & $\begin{array}{l}\text { Surveys were distributed to } \\
\text { EMS providers who attended } \\
\text { an educational class }\end{array}$ & $\begin{array}{l}\text { - } 74 \% \text { had heard of a naloxone program } \\
-84 / 154(56 \%) \text { did not view naloxone } \\
\text { training as effective in reducing deaths } \\
-96 / 154(64 \%) \text { reported no interest in } \\
\text { participating in training drug users; } \\
\text { however, } 47 / 154(30 \%) \text { reported interest } \\
\text { in a class about addiction and health } \\
\text { issues education } \\
\text { - Barriers: dirty needles, naloxone theft, } \\
\text { administration and other acute health } \\
\text { factors were raised }\end{array}$ & $\begin{array}{l}\text { National } \\
\text { Institute on } \\
\text { Drug Abuse }\end{array}$ \\
\hline $\begin{array}{l}\text { Winstanley et al., } \\
2016 \\
\text { Barriers to implementation of } \\
\text { opioid overdose prevention } \\
\text { programs in Ohio. Substance } \\
\text { Abuse [73] }\end{array}$ & US & $\begin{array}{l}\text { Cross-sectional survey with } \\
\text { quantitative and qualitative } \\
\text { questions }\end{array}$ & $\begin{array}{c}\mathrm{N}=20 \text { OOPP site } \\
\text { representatives } \\
47.8 \% \text { were in suburban } \\
\text { countries, } 34.8 \% \text { in urban } \\
\text { countries and } 17.4 \% \text { in } \\
\text { rural communities }\end{array}$ & $\begin{array}{l}\text { Contact person of opioid } \\
\text { overdose prevention programs } \\
\text { (OOPP) were given a survey } \\
\text { about their program } \\
\text { Initial list from the Ohio } \\
\text { Department of Health } \\
\text { Survey was emailed or given } \\
\text { over the phone }\end{array}$ & $\begin{array}{l}-15 / 20(83 \%) \text { of programs experience } \\
\text { barriers, which were categorized into: } \\
\text { stigma }(n=14) \text {, costs }(n=7) \text {, staffing }(n \\
=5) \text {, legal }(n=4) \text {, regulatory }(n=3) \text { and } \\
\text { clients }(n=3) \text {. }\end{array}$ & None stated \\
\hline
\end{tabular}


Table A3. Cont.

\begin{tabular}{|c|c|c|c|c|c|c|}
\hline \multicolumn{7}{|c|}{ Professionals' Perspective } \\
\hline Author, Title and Journal & Country & Data Collection Method & Participants & Interventions/Study Details & Outcomes & $\begin{array}{c}\text { Funding/ } \\
\text { Sponsorship }\end{array}$ \\
\hline $\begin{array}{c}\text { Zaller et al., } \\
2013 \\
\text { The feasibility of } \\
\text { pharmacy-based naloxone } \\
\text { distribution interventions: a } \\
\text { qualitative study with injection } \\
\text { drug users and pharmacy staff } \\
\text { in Rhode Island. } \\
\text { Substance Use and Misuse [74] }\end{array}$ & US & Qualitative interviews & $\begin{array}{c}\mathrm{N}=21 \text { pharmacy staff } \\
\text { Median age } 35,80 \% \text { white, } \\
73.3 \% \text { male } \\
\mathrm{N}=21 \text { people who inject } \\
\text { drugs } \\
\text { Median age } 36 \text { and } 71 \% \\
\text { white, } 66 \% \text { male }\end{array}$ & $\begin{array}{l}\text { People who inject drugs were } \\
\text { recruited from syringe } \\
\text { exchange programs and } \\
\text { recognized community } \\
\text { locations } \\
\text { Pharmacy staff were recruited } \\
\text { through in-person visits or } \\
\text { phone calls } \\
\text { Interviews lasted } 45 \text { min to } 1.5 \\
\mathrm{~h}\end{array}$ & $\begin{array}{l}\text { - Pharmacists were not aware of the } \\
\text { prevalence of overdose and all IDUs } \\
\text { reported experiencing or witnessing an } \\
\text { overdose } \\
\text { - Positive and negative attitudes toward } \\
\text { naloxone use from injecting drug users } \\
\text { - Pharmacy staff were concerned about } \\
\text { increase in risk behaviours } \\
\text { - Overall, many IDUs and pharmacy staff } \\
\text { were supportive } \\
\text { - Both groups misjudged each other's } \\
\text { willingness to participate in a naloxone } \\
\text { intervention }\end{array}$ & None stated \\
\hline
\end{tabular}

ED = Emergency Department, EMS = Emergency Medical Services, IM = Intramuscular, IN = Intranasal, IDU/s = Injecting Drug Use/Users, MD = Medical doctor, NHS = National Health Service, NP = Nurse Practitioner, OOPP = Opioid Overdose Prevention Programs, OST = Opioid Substitution Therapy, SWOT = Strength Weakness Opportunities Threats, THN = Take-Home Naloxone, UK = United Kingdom, US = United States. 


\section{References}

1. Roxburgh, A.; Ritter, A.; Slade, T.; Burns, L. Trends in Drug Use and Related Harms in Australia, 2001 to 2013; National Drug and Alcohol Research Centre, University of New South Wales: Sydney, Australia, 2013; Available online: https://ndarc.med.unsw.edu.au/RESOURCE/TRENDS-DRUG-USE-AND-RELATEDHARMS-AUSTRALIA (accessed on 8 June 2020).

2. Roxburgh, A.; Burns, L. Accidental Drug-Induced Deaths Due to Opioids in Australia, 2011; National Drug and Alcohol Research Centre: Sydney, Australia, 2015; Available online: https://ndarc.med.unsw.edu.au/resource/ accidental-drug-induced-deaths-due-opioids-australia-2011 (accessed on 9 June 2020).

3. Bureau of Infrastructure, Transport and Regional Economics (BITRE). Road Deaths Australia, 2012 Statistical Summary; Department of Infrastructure and Transport: Canberra, Australia, 2013. Available online: https://bitre.gov.au/publications/ongoing/files/RDA_Summary_2012_June.pdf (accessed on 9 June 2020).

4. Rintoul, A.C.; Dobbin, D.M.; Drummer, O.H.; Ozanne-Smith, J. Increasing deaths involving oxycodone, Victoria, Australia, 2000-2009. Int. J. Prev. 2011, 17, 254.

5. Blanch, B.; Pearson, S.A.; Haber, P.S. An overview of the patterns of prescription opioid use, costs and related harms in Australia. Br. J. Clin. Pharmacol. 2014, 78, 1159-1166. [CrossRef] [PubMed]

6. Roxburgh, A.; Bruno, R.; Larance, B.; Burns, L. Prescription of opioid analgesics and related harms in Australia. Med. J. Aust. 2011, 195, 280-284. [CrossRef]

7. Noble, M.; Treadwell, J.R.; Tregear, S.J.; Coates, V.H.; Wiffen, P.J.; Akafomo, C.; Schoelles, K.M.; Chou, R.; Erskine, A. Long-term opioid management for chronic noncancer pain. Cochrane Database Syst. Rev. 2010, 2010, 006605. [CrossRef] [PubMed]

8. Juurlink, D.N.; Dhalla, I.A. Dependence and Addiction During Chronic Opioid Therapy. J. Med. Toxicol. 2012, 8, 393-399. [CrossRef] [PubMed]

9. Weiss, R.D.; Potter, J.S.; Griffin, M.L.; McHugh, R.K.; Haller, D.; Jacobs, P.; Gardin, J.; Fischer, D.; Rosen, K.D. Reasons for opioid use among patients with dependence on prescription opioids: The role of chronic pain. J. Subst. Abus. Treat. 2014, 47, 140-145. [CrossRef]

10. Zgierska, A.; Miller, M.; Rabago, D. Patient satisfaction, prescription drug abuse, and potential unintended consequences. JAMA 2012, 307, 1377-1378. [PubMed]

11. Van Zee, A. The Promotion and Marketing of Oxycontin: Commercial Triumph, Public Health Tragedy. Am. J. Public Health 2009, 99, 221-227. [CrossRef] [PubMed]

12. Cerdá, M.; Ransome, Y.; Keyes, K.M.; Koenen, K.C.; Tardiff, K.; Vlahov, D.; Galea, S. Revisiting the role of the urban environment in substance use: The case of analgesic overdose fatalities. Am. J. Public Health 2013, 103, 2252-2260. [CrossRef]

13. Riggs, C.S.; Billups, S.J.; Flores, S.; Patel, R.J.; Heilmann, R.M.; Milchak, J.L. Opioid Use for Pain Management After Implementation of a Medicaid Short-Acting Opioid Quantity Limit. J. Manag. Care Spec. Pharm. 2017, 23, 346-354. [CrossRef]

14. Stafford, J.; Breen, C. Australian Drug Trends 2016. Findings from the Illicit Drug Reporting System (IDRS); National Drug and Alcohol Research Centre, University of New South Wales: Sydney, Australia, 2017; Available online: https://ndarc.med.unsw.edu.au/sites/default/files/ndarc/resources/national-idrs_2016_ finalwith-customs.pdf (accessed on 9 June 2020).

15. Häuser, W.; Schug, S.; Furlan, A.D. The opioid epidemic and national guidelines for opioid therapy for chronic noncancer pain: A perspective from different continents. PAIN Rep. 2017, 2, e599.

16. Wermeling, D.P. Review of naloxone safety for opioid overdose: Practical considerations for new technology and expanded public access. Adv. Drug Saf. 2015, 6, 20-31. [CrossRef] [PubMed]

17. Robinson, A.; Wermeling, D.P. Intranasal naloxone administration for treatment of opioid overdose. Am. J. Health Syst. Pharm. 2014, 71, 2129-2135. [CrossRef] [PubMed]

18. Freeman, P.R.; Goodin, A.; Troske, S.; Strahl, A.; Fallin, A.; Green, T.C. Pharmacists role in opioid overdose: Kentucky pharmacists willingness to participate in naloxone dispensing. J. Am. Pharm. Assoc. 2003, 57, S28-S33. [CrossRef]

19. Weaver, L.; Palombi, L.; Bastianelli, K.M. Naloxone Administration for Opioid Overdose Reversal in the Prehospital Setting: Implications for Pharmacists. J. Pharm. Pract. 2017, 31, 91-98. [CrossRef]

20. He, F.; Jiang, Y.; Li, L. The effect of naloxone treatment on opioid-induced side effects: A meta-analysis of randomized and controlled trails. Medicine 2016, 95, e4729. [CrossRef] 
21. Public Health England. Widening the Availability of Naloxone; Department of Health: England, UK, 2015. Available online: https:/www.gov.uk/government/publications/widening-the-availability-of-naloxone/ widening-the-availability-of-naloxone (accessed on 9 June 2020).

22. Galea, S.; Worthington, N.; Piper, T.M.; Nandi, V.V.; Curtis, M.; Rosenthal, D.M. Provision of naloxone to injection drug users as an overdose prevention strategy: Early evidence from a pilot study in New York City. Addict. Behav 2006, 31, 907-912. [CrossRef]

23. Lenton, S.R.; Dietze, P.M.; Jauncey, M. Australia reschedules naloxone for opioid overdose. Med. J. Aust. 2016, 204, 146-147. [CrossRef]

24. College of Pharmacists of British Columbia. Non-Prescrition Naloxone Now Available Outside of Pharmacy; College of Pharmacists of British Columbia: Vancouver, BC, Canada, 2016; Available online: http:// www.bcpharmacists.org/news/non-prescription-naloxone-now-available-outside-pharmacies (accessed on 9 June 2020).

25. Therapeutic Goods Administration (TGA). Scheduling Delegate's Final Decisions: ACMS, November 2015; Department of Health: Sydney, Australia, 2015. Available online: https://www.tga.gov.au/book-page/partfinal-decisions-matters-referred-expert-advisory-committee-11-14\#nalox (accessed on 9 June 2020).

26. Olsen, A.; McDonald, D.; Lenton, S.; Dietze, P. Independent Evaluation of the 'Implementing Expanded, Naloxone Availability in the ACT (I-ENAACT) Program', 2011-2014, Final Report; Centre for Research Into Injecting Drug Use: Melbourne, Australia, 2015; Available online: http://www.atoda.org.au/wp-content/uploads/NaloxoneEvaluation-Report-FINAL_August-2015_BI.pdf (accessed on 9 June 2020).

27. Arksey, H.; O'Malley, L. Scoping studies: Towards a methodological framework. Int. J. Soc. Res. Methodol. 2005, 8, 19-32. [CrossRef]

28. Moher, D.; Liberati, A.; Tetzlaff, J.; Altman, D.G.; The PRISMA Group. Preferred Reporting Items for Systematic Reviews and Meta-Analyses: The PRISMA Statement. PLoS Med. 2009, 6, e1000097. [CrossRef]

29. Bagley, S.M.; Peterson, J.; Cheng, D.M.; Jose, C.; Quinn, E.; O'Connor, P.G.; Walley, A.Y. Overdose Education and Naloxone Rescue Kits for Family Members of Individuals Who Use Opioids: Characteristics, Motivations, and Naloxone Use. Subst. Abus. 2015, 36, 149-154. [CrossRef] [PubMed]

30. Bennett, A.S.; Bell, A.; Tomedi, L.; Hulsey, E.G.; Kral, A.H. Characteristics of an overdose prevention, response, and naloxone distribution program in Pittsburgh and Allegheny County, Pennsylvania. J. Urban Health 2011, 88, 1020-1030. [CrossRef] [PubMed]

31. Bird, S.M.; McAuley, A.; Perry, S.; Hunter, C. Effectiveness of Scotland's National Naloxone Programme for reducing opioid-related deaths: A before (2006-10) versus after (2011-13) comparison. Addiction 2016, 111, 883-891. [CrossRef] [PubMed]

32. Doe-Simkins, M.; Quinn, E.; Xuan, Z.; Sorensen-Alawad, A.; Hackman, H.; Ozonoff, A.; Walley, A.Y. Overdose rescues by trained and untrained participants and change in opioid use among substance-using participants in overdose education and naloxone distribution programs: A retrospective cohort study. BMC Public Health 2014, 14, 297. [CrossRef] [PubMed]

33. Dong, K.A.; Taylor, M.; Wild, C.T.; Villa-Roel, C.; Rose, M.; Salvalaggio, G.; Rowe, B.H. Community-based naloxone: A Canadian pilot program. Can. J. Addict. Med. 2012, 3, 4-9.

34. Enteen, L.; Bauer, J.; McLean, R.; Wheeler, E.; Huriaux, E.; Kral, A.H.; Bamberger, J.D. Overdose prevention and naloxone prescription for opioid users in San Francisco. J. Urban Health 2010, 87, 931-941. [CrossRef] [PubMed]

35. Green, T.C.; Heimer, R.; Grau, L.E. Distinguishing signs of opioid overdose and indication for naloxone: An evaluation of six overdose training and naloxone distribution programs in the United States. Addiction 2008, 103, 979-989. [CrossRef]

36. Jones, J.D.; Roux, P.; Stancliff, S.; Matthews, W.; Comer, S.D. Brief overdose education can significantly increase accurate recognition of opioid overdose among heroin users. Int. J. Drug Policy 2014, 25, 166-170. [CrossRef]

37. Lankenau, S.E.; Wagner, K.D.; Silva, K.; Kecojevic, A.; Iverson, E.; McNeely, M.; Kral, A.H. Injection drug users trained by overdose prevention programs: Responses to witnessed overdoses. J. Community Health 2013, 38, 133-141. [CrossRef]

38. Leece, P.N.; Hopkins, S.; Marshall, C.; Orkin, A.; Gassanov, M.A.; Shahin, R.M. Development and implementation of an opioid overdose prevention and response program in Toronto, Ontario. Can. J. Public Health 2013, 104, e200-e204. [CrossRef] 
39. Lott, D.C.; Rhodes, J. Opioid overdose and naloxone education in a substance use disorder treatment program. Am. J. Addict. 2016, 25, 221-226. [CrossRef] [PubMed]

40. Madah-Amiri, D.; Clausen, T.; Lobmaier, P. Rapid widespread distribution of intranasal naloxone for overdose prevention. Drug Alcohol. Depend. 2017, 173, 17-23. [CrossRef] [PubMed]

41. Maxwell, S.; Bigg, D.; Stanczykiewicz, K.; Carlberg-Racich, S. Prescribing naloxone to actively injecting heroin users: A program to reduce heroin overdose deaths. J. Addict. Dis. 2006, 25, 89-96. [CrossRef]

42. Piper, T.M.; Stancliff, S.; Rudenstine, S.; Sherman, S.; Nandi, V.; Clear, A.; Galea, S. Evaluation of a naloxone distribution and administration program in New York City. Subst. Use Misuse 2008, 43, 858-870. [CrossRef] [PubMed]

43. Rowe, C.; Santos, G.M.; Vittinghoff, E.; Wheeler, E.; Davidson, P.; Coffin, P.O. Predictors of participant engagement and naloxone utilization in a community-based naloxone distribution program. Addiction 2015, 110, 1301-1310. [CrossRef] [PubMed]

44. Seal, K.H.; Thawley, R.; Gee, L.; Bamberger, J.; Kral, A.H.; Ciccarone, D.; Downing, M.; Edlin, B.R. Naloxone distribution and cardiopulmonary resuscitation training for injection drug users to prevent heroin overdose death: A pilot intervention study. J. Urban Health 2005, 82, 303-311. [CrossRef]

45. Sherman, S.G.; Gann, D.S.; Scott, G.; Carlberg, S.; Bigg, D.; Heimer, R. A qualitative study of overdose responses among Chicago IDUs. Harm Reduct. J. 2008, 5, 2. [CrossRef]

46. Strang, J.; Manning, V.; Mayet, S.; Best, D.L.; Titherington, E.; Santana, L.; Offor, E.; Semmler, C. Overdose training and take-home naloxone for opiate users: Prospective cohort study of impact on knowledge and attitudes and subsequent management of overdoses. Addiction 2008, 103, 1648-1657. [CrossRef]

47. Tobin, K.E.; Sherman, S.G.; Beilenson, P.; Welsh, C.; Latkin, C.A. Evaluation of the Staying Alive programme: Training injection drug users to properly administer naloxone and save lives. Int. J. Drug Policy 2009, 20, 131-136. [CrossRef]

48. Wagner, K.D.; Valente, T.W.; Casanova, M.; Partovi, S.M.; Mendenhall, B.M.; Hundley, J.H.; Gonzalez, M.; Unger, J.B. Evaluation of an overdose prevention and response training programme for injection drug users in the Skid Row area of Los Angeles, CA. Int. J. Drug Policy 2010, 21, 186-193. [CrossRef]

49. Walley, A.Y.; Xuan, Z.; Hackman, H.H.; Quinn, E.; Doe-Simkins, M.; Sorensen-Alawad, A.; Ruiz, S.; Ozonoff, A. Opioid overdose rates and implementation of overdose education and nasal naloxone distribution in Massachusetts: Interrupted time series analysis. BMJ 2013, 346, f174. [CrossRef]

50. Williams, A.V.; Marsden, J.; Strang, J. Training family members to manage heroin overdose and administer naloxone: Randomized trial of effects on knowledge and attitudes. Addiction 2014, 109, 250-259. [CrossRef]

51. Yokell, M.A.; Green, T.C.; Bowman, S.; McKenzie, M.; Rich, J.D. Opioid overdose prevention and naloxone distribution in Rhode Island. Med. Health 2011, 94, 240-242.

52. Mueller, S.R.; Koester, S.; Glanz, J.M.; Gardner, E.M.; Binswanger, I.A. Attitudes Toward Naloxone Prescribing in Clinical Settings: A Qualitative Study of Patients Prescribed High Dose Opioids for Chronic Non-Cancer Pain. J. Gen. Int. Med. 2017, 32, 277-283. [CrossRef]

53. Oliva, E.M.; Nevedal, A.; Lewis, E.T.; McCaa, M.D.; Cochran, M.F.; Konicki, P.E.; Davis, C.S.; Wilder, C. Patient perspectives on an opioid overdose education and naloxone distribution program in the U.S. Department of Veterans Affairs. Subst. Abus. 2016, 37, 118-126. [CrossRef]

54. Worthington, N.; Piper, T.M.; Galea, S.; Rosenthal, D. Opiate users' knowledge about overdose prevention and naloxone in New York City: A focus group study. Harm Reduct. J. 2006, 3, 19. [CrossRef]

55. Wright, N.; Oldham, N.; Francis, K.; Jones, L. Homeless drug users' awareness and risk perception of peer "take home naloxone" use-a qualitative study. Subst. Abus. Treat. Prev. Policy 2006, 1, 28. [CrossRef]

56. Delaney, W.; Huff, J.; Mini, S.; Thomas, A.; Tremaglio, R. Coprescribing naloxone for patients on chronic opioid therapy: Lessons learned from a patient-safety initiative in primary care training sites. J. Opioid Manag. 2016, 12, 360-366. [CrossRef]

57. Strang, J.; Powis, B.; Best, D.; Vingoe, L.; Griffiths, P.; Taylor, C.; Welch, S.; Gossop, M. Preventing opiate overdose fatalities with take-home naloxone: Pre-launch study of possible impact and acceptability. Addiction 1999, 94, 199-204. [CrossRef]

58. Bachhuber, M.A.; McGinty, E.E.; Kennedy-Hendricks, A.; Niederdeppe, J.; Barry, C.L. Messaging to increase public support for naloxone distribution policies in the United States: Results from a randomized survey experiment. PLoS ONE 2015, 10, e0130050. [CrossRef] 
59. Barocas, J.A.; Baker, L.; Hull, S.J.; Stokes, S.; Westergaard, R.P. High uptake of naloxone-based overdose prevention training among previously incarcerated syringe-exchange program participants. Drug Alcohol. Depend. 2015, 154, 283-286. [CrossRef] [PubMed]

60. Kestler, A.; Buxton, J.; Meckling, G.; Giesler, A.; Lee, M.; Fuller, K.; Quian, H.; Marks, D.; Scheuermeyer, F.X. Factors Associated with Participation in an Emergency Department-Based Take-Home Naloxone Program for At-Risk Opioid Users. Ann. Emerg. Med. 2017, 69, 340-346. [CrossRef] [PubMed]

61. McAuley, A.; Munro, A.; Bird, S.M.; Hutchinson, S.J.; Goldberg, D.J.; Taylor, A. Engagement in a National Naloxone Programme among people who inject drugs. Drug Alcohol. Depend. 2016, 162, 236-240. [CrossRef] [PubMed]

62. Rowe, C.; Santos, G.M.; Vittinghoff, E.; Wheeler, E.; Davidson, P.; Coffin, P.O. Neighborhood-Level and Spatial Characteristics Associated with Lay Naloxone Reversal Events and Opioid Overdose Deaths. J. Urban Health 2016, 93, 117-130. [CrossRef]

63. Seal, K.H.; Downing, M.; Kral, A.H.; Singleton-Banks, S.; Hammond, J.-P.; Lorvick, J.; Ciccarone, D.; Edlin, B.R. Attitudes about prescribing take-home naloxone to injection drug users for the management of heroin overdose: A survey of street-recruited injectors in the San Francisco Bay Area. J. Urban Health 2003, 80, 291-301. [CrossRef]

64. Bailey, A.M.; Wermeling, D.P. Naloxone for Opioid Overdose Prevention: Pharmacists' Role in Community-Based Practice Settings. Ann. Pharm. 2014, 48, 601-606. [CrossRef]

65. Behar, E.; Rowe, C.; Santos, G.M.; Coffa, D.; Turner, C.; Santos, N.C.; Coffin, P.O. Acceptability of Naloxone Co-Prescription Among Primary Care Providers Treating Patients on Long-Term Opioid Therapy for Pain. J. Gen. Intern. Med. 2017, 32, 291-295. [CrossRef]

66. Drainoni, M.-L.; Koppelman, E.A.; Feldman, J.A.; Walley, A.Y.; Mitchell, P.M.; Ellison, J.; Bernstein, E. Why is it so hard to implement change? A qualitative examination of barriers and facilitators to distribution of naloxone for overdose prevention in a safety net environment. BMC Res. Notes 2016, 9, 465. [CrossRef]

67. Gatewood, A.K.; Van Wert, M.J.; Andrada, A.P.; Surkan, P.J. Academic physicians' and medical students' perceived barriers toward bystander administered naloxone as an overdose prevention strategy. Addict. Behav. 2016, 61, 40-46. [CrossRef]

68. Green, T.C.; Bowman, S.E.; Zaller, N.D.; Ray, M.; Case, P.; Heimer, R. Barriers to medical provider support for prescription naloxone as overdose antidote for lay responders. Subst. Use Misuse 2013, 48, 558-567. [CrossRef]

69. Leece, P.; Orkin, A.; Shahin, R.; Steele, L.S. Can naloxone prescription and overdose training for opioid users work in family practice? Perspectives of family physicians. Can. Fam. Physician 2015, 61, 538-543. [PubMed]

70. Nielsen, S.; Menon, N.; Larney, S.; Farrell, M.; Degenhardt, L. Community pharmacist knowledge, attitudes and confidence regarding naloxone for overdose reversal. Addiction 2016, 111, 2177-2186. [CrossRef] [PubMed]

71. Sondhi, A.; Ryan, G.; Day, E. Stakeholder perceptions and operational barriers in the training and distribution of take-home naloxone within prisons in England. Harm Reduct. J. 2016, 13, 5. [CrossRef] [PubMed]

72. Tobin, K.E.; Gaasch, W.R.; Clarke, C.; MacKenzie, E.; Latkin, C.A. Attitudes of Emergency Medical Service providers towards naloxone distribution programs. J. Urban Health 2005, 82, 296-302. [CrossRef]

73. Winstanley, E.L.; Clark, A.; Feinberg, J.; Wilder, C.M. Barriers to Implementation of Opioid Overdose Prevention Programs in Ohio. Subst. Abus. 2016, 37, 42-46. [CrossRef]

74. Zaller, N.D.; Yokell, M.A.; Green, T.C.; Gaggin, J.; Case, P. The feasibility of pharmacy-based naloxone distribution interventions: A qualitative study with injection drug users and pharmacy staff in Rhode Island. Subst. Use Misuse 2013, 48, 590-599. [CrossRef]

75. Ashrafioun, L.; Gamble, S.; Herrmann, M.; Baciewicz, G. Evaluation of knowledge and confidence following opioid overdose prevention training: A comparison of types of training participants and naloxone administration methods. Subst. Abus. 2016, 37, 76-81. [CrossRef]

76. Dahlem, C.H.; Horstman, M.J.; Williams, B.C. Development and implementation of intranasal naloxone opioid overdose response protocol at a homeless health clinic. J. Am. Assoc. Nurse Pract. 2016, 28, 11-18. [CrossRef]

77. Ministerial Council on Drug Strategy. National Drug Strategy 2010-2015; Australian Government: Sydney, Australia, 2011. Available online: http://www.nationaldrugstrategy.gov.au/internet/drugstrategy/publishing. nsf/Content/home (accessed on 9 June 2020). 
78. Gilles, S.M. Needle exchange programs: Factors that influence attitudes and local policy. Theses Diss. 1994, 267,5 .

79. White, B.; Haber, P.S.; Day, C.A. Community attitudes towards harm reduction services and a newly established needle and syringe automatic dispensing machine in an inner-city area of Sydney, Australia. Int. J. Drug Policy 2016, 27, 121-126. [CrossRef]

80. American Foundation for Aids Research. Public Safety, Law Enforcement and Syringe Exchange; The Foundation for AIDS Research: New York, NY, USA, 2013; Available online: http://www.amfar.org/Search/?q=fact+sheet (accessed on 9 June 2020).

81. Dolan, K.; MacDonald, M.; Silins, E.; Topp, L. Needle and Syringe Programs: A Review of the Evidence; Australian Government Department of Health and Ageing: Canberra, Australia, 2005. Available online: https:/www.health.gov.au/internet/main/publishing.nsf/content/ 3AAED699516CE2DCA257BF0001E7255/\$File/evid.pdf (accessed on 30 May 2020).

82. Fromer, L. Prevention of Anaphylaxis: The Role of the Epinephrine Auto-Injector. Am. J. Med. 2016, 129, 1244-1250. [CrossRef] [PubMed]

83. Van Boekel, L.C.; Brouwers, E.P.; van Weeghel, J.; Garretsen, H.F. Stigma among health professionals towards patients with substance use disorders and its consequences for healthcare delivery: Systematic review. Drug Alcohol. Depend. 2013, 131, 23-35. [CrossRef] [PubMed]

84. Medical Board of Australia. Good Medical Practice: A Code of Conduct for Doctors in Australia; Australian Health Practitioner Regulatory Agency: Sydney, Australia, 2014. Available online: http://www.medicalboard. gov.au/Codes-Guidelines-Policies/Code-of-conduct.aspx (accessed on 2 June 2020).

85. Pharmacy Board of Australia. For Pharmacists-Code of Conduct; Australian Health Practitioner Regulatory Agency: Sydney, Australian, 2014. Available online: http://www.pharmacyboard.gov.au/Codes-Guidelines/ Code-of-conduct.aspx (accessed on 2 June 2020).

86. Centers for Disease Control and Prevention (CDC). Prescription Opioid Overdose Data; US Department of Health and Human Services and Prevention: Atlanta, GA, USA, 2016. Available online: https://www.cdc. gov/drugoverdose/data/overdosehtml (accessed on 2 June 2020).

87. Volkow, N.D.; McLellan, A.T. Opioid Abuse in Chronic Pain- Misconceptions and Mitigation Strategies. N. Engl. J. Med. 2016, 374, 1253-1263. [CrossRef]

88. Coe, M.A.; Walsh, S.L. Distribution of Naloxone for Overdose Prevention to Chronic Pain Patients. Prev. Med. 2015, 80, 41-43. [CrossRef]

89. Coffin, P.O.; Behar, E.; Rowe, C.; Santos, G.-M.; Coffa, D.; Bald, M.; Vittinghoff, E. Non-randomized intervention study of naloxone coprescription for primary care patients receiving long-term opioid therapy for pain. Ann. Int. Med. 2016, 165, 245-252. [CrossRef] [PubMed]

90. Ward, J.; Hall, W.; Mattick, R.P. Role of maintenance treatment in opioid dependence. Lancet 1999, 353, 221-226. [CrossRef]

91. McArthur, M. A History of Methadone Treatment in Australia: The Influence of Social Control Arguments in Its Development; History of Crime, Policing and Punishment Conference Canberra: Canberra, Australia, 1999; Available online: http://www.aic.gov.au/media_library/conferences/hcpp/mcarthur.pdf (accessed on 5 June 2020).

92. Beletsky, L.; Ruthazer, R.; Macalino, G.E.; Rich, J.D.; Tan, L.; Burris, S. Physicians' knowledge of and willingness to prescribe naloxone to reverse accidental opiate overdose: Challenges and opportunities. J. Urban Health 2007, 84, 126-136. [CrossRef]

93. Scales, D.C.; Fischer, H.D.; Li, P.; Bierman, A.S.; Fernandes, O.; Mamdani, M.; Rochon, P.; Urbach, D.R.; Bell, C.M. Unintentional Continuation of Medications Intended for Acute Illness After Hospital Discharge: A Population-Based Cohort Study. J. Gen. Int. Med. 2016, 31, 196-202. [CrossRef]

94. Davis, C.S.; Carr, D. Legal changes to increase access to naloxone for opioid overdose reversal in the United States. Drug Alcohol. Depend. 2015, 157, 112-120. [CrossRef] 
95. Hakansson, A.; Vedin, A.; Wallin, C.; Kral, A.H. Distribution of naloxone to prevent death from heroin overdose. Study of opioid dependent patients' attitudes to be part of the antidote program. Lakartidningen 2013, 110, 1340-1342.

96. Coffin, P.O.; Fuller, C.; Vadnai, L.; Blaney, S.; Galea, S.; Vlahov, D. Preliminary Evidence of Health Care Provider Support for Naloxone Prescription as Overdose Fatality Prevention Strategy in New York City. J. Urban Health 2003, 80, 288-290. [CrossRef] [PubMed]

Publisher's Note: MDPI stays neutral with regard to jurisdictional claims in published maps and institutional affiliations.

(C) 2020 by the authors. Licensee MDPI, Basel, Switzerland. This article is an open access article distributed under the terms and conditions of the Creative Commons Attribution (CC BY) license (http://creativecommons.org/licenses/by/4.0/). 